\title{
Moduli spaces and braid monodromy types of bidouble covers of the quadric
}

\author{
FABRIZIO CATANESE \\ MICHAEL LÖNNE \\ BRONISLAW WAJNRYB
}

Bidouble covers $\pi: S \rightarrow Q:=\mathbb{P}^{1} \times \mathbb{P}^{1}$ of the quadric are parametrized by connected families depending on four positive integers $a, b, c, d$. In the special case where $b=d$ we call them $a b c$-surfaces.

Such a Galois covering $\pi$ admits a small perturbation yielding a general 4-tuple covering of $Q$ with branch curve $\Delta$, and a natural Lefschetz fibration obtained from a small perturbation of the composition $p_{1} \circ \pi$.

We prove a more general result implying that the braid monodromy factorization corresponding to $\Delta$ determines the three integers $a, b, c$ in the case of $a b c$-surfaces. We introduce a new method in order to distinguish factorizations which are not stably equivalent.

This result is in sharp contrast with a previous result of the first and third author, showing that the mapping class group factorizations corresponding to the respective natural Lefschetz pencils are equivalent for $a b c$-surfaces with the same values of $a+c, b$. This result hints at the possibility that $a b c$-surfaces with fixed values of $a+c, b$, although diffeomorphic but not deformation equivalent, might be not canonically symplectomorphic.

14J15; 14J29, 14J80, 14D05, 53D05, 57R50

\section{Introduction}

Bidouble covers of the quadric are smooth projective complex surfaces $S$ endowed with a (finite) Galois covering $\pi: S \rightarrow Q:=\mathbb{P}^{1} \times \mathbb{P}^{1}$ with Galois group $(\mathbb{Z} / 2 \mathbb{Z})^{2}$.

More concretely, they are defined by a single pair of equations

$$
\begin{aligned}
z^{2} & =f_{(2 a, 2 b)}\left(x_{0}, x_{1} ; y_{0}, y_{1}\right) \\
w^{2} & =g_{(2 c, 2 d)}\left(x_{0}, x_{1} ; y_{0}, y_{1}\right)
\end{aligned}
$$


where we shall assume for simplicity that $a, b, c, d \in \mathbb{N}_{\geq 3}$, and the notation $f_{(2 a, 2 b)}$ denotes that $f$ is a bihomogeneous polynomial, homogeneous of degree $2 a$ in the variables $x$ and of degree $2 b$ in the variables $y$.

These surfaces are simply connected and minimal of general type, and they were introduced by the first author [5] in order to show that the moduli spaces $\mathfrak{M}_{\chi, K^{2}}$ of smooth minimal surfaces of general type $S$ with $\chi(S):=\chi\left(\mathcal{O}_{S}\right)=\chi, K_{S}^{2}=K^{2}$, need not be equidimensional or irreducible. (The study of the corresponding closed components of the moduli space was pursued by the first author [6] and by Manetti [24; 25].)

Given in fact our four integers $a, b, c, d \in \mathbb{N}_{\geq 3}$, considering the so-called natural deformations of these bidouble covers, defined by equations ${ }^{1}$

$$
\begin{aligned}
z^{2} & =f_{(2 a, 2 b)}\left(x_{0}, x_{1} ; y_{0}, y_{1}\right)+w \Phi_{(2 a-c, 2 b-d)}\left(x_{0}, x_{1} ; y_{0}, y_{1}\right) \\
w^{2} & =g_{(2 c, 2 d)}\left(x_{0}, x_{1} ; y_{0}, y_{1}\right)+z \Psi_{(2 c-a, 2 d-b)}\left(x_{0}, x_{1} ; y_{0}, y_{1}\right)
\end{aligned}
$$

one defines a bigger open subset $\mathfrak{N}_{a, b, c, d}^{\prime}$ of the moduli space, whose closure $\overline{\mathfrak{N}}_{a, b, c, d}$ is an irreducible component of $\mathfrak{M}_{\chi, K^{2}}$, where

$$
\chi=1+(a-1)(b-1)+(c-1)(d-1)+(a+c-1)(b+d-1)
$$

and

$$
K^{2}=8(a+c-2)(b+d-2) .
$$

In general our knowledge about the moduli spaces $\mathfrak{M}_{\chi, K^{2}}$ is rather scanty, even if we make the drastic restriction only to consider the subset $\mathfrak{M}_{\chi, K^{2}}^{00}$ corresponding to isomorphism classes $[S]$ of simply connected minimal surfaces. $\mathfrak{M}_{\chi, K^{2}}$ is a quasiprojective variety, with a finite number of connected components, and $\mathfrak{M}_{\chi, K^{2}}^{00}$ is a union of connected components of $\mathfrak{M}_{\chi, K^{2}}$.

For a surface $S$ with $[S] \in \mathfrak{M}_{\chi}^{00}, K^{2}$ there are (by Freedman's theorem [16]) at most two topological types, according to the parity of the intersection form $q_{S}: H^{2}(S, \mathbb{Z}) \rightarrow \mathbb{Z}$. If $q_{S}$ is even (ie, $\operatorname{Im}\left(q_{S}\right) \subset 2 \mathbb{Z}$ ) then $S$ is orientedly homeomorphic to a connected sum of copies of $\mathbb{P}^{1} \times \mathbb{P}^{1}$ and copies of a K3 surface $Y$ (possibly taken with reversed orientation); if $q_{S}$ is odd $\left(\operatorname{Im}\left(q_{S}\right) \not \subset 2 \mathbb{Z}\right)$ then $S$ is orientedly homeomorphic to a connected sum of copies of $\mathbb{P}^{2}$ and copies of $\mathbb{P}^{2}$ with reversed orientation.

Thus the topology of $S$ does not give sufficient information in order to distinguish the connected components $\mathfrak{N} \subset \mathfrak{M}_{\chi, K^{2}}^{00}$. Note that if $\mathfrak{N}$ is such a connected component and $S, S^{\prime}$ are minimal surfaces with $[S],\left[S^{\prime}\right] \in \mathfrak{N}$ (we then simply say that $S, S^{\prime}$ have the same deformation type), then, by the classical theorem of Ehresmann [15], there exists an orientation preserving diffeomorphism $\Psi: S^{\prime} \rightarrow S$ carrying the canonical class $c_{1}\left(K_{S}\right)$ to the canonical class $c_{1}\left(K_{S^{\prime}}\right)$.

\footnotetext{
${ }^{1}$ In the following formula, a polynomial of negative degree is identically zero.
} 
In [7] (see also [9]) the first author showed that to each such surface $S$ one can associate a symplectic manifold $(S, \omega)$, unique up to symplectomorphism, and such that the de Rham class of $\omega$ equals $c_{1}\left(K_{S}\right)$. Moreover, it was shown that this symplectomorphism class, called "canonical symplectomorphism class", is an invariant of the connected component $\mathfrak{N}$.

The so-called Manetti surfaces yield examples (see Manetti [26] and Catanese [9; 8]) of surfaces $S, S^{\prime}$ lying in distinct connected components of $\mathfrak{M}_{\chi, K^{2}}$ but having the same canonical symplectomorphism class.

Whether this also occurs for $\mathfrak{M}_{\chi, K^{2}}^{00}$ is an interesting open question, especially motivated by the results of Catanese and Wajnryb [12], where the families $\mathfrak{N}_{a, b, c}$ of the so-called $a b c$-surfaces, essentially a partial closure of $\mathfrak{N}_{a, b, c, b}^{\prime}$, were shown to provide examples of simply connected surfaces $S, S^{\prime}$ belonging to distinct connected components $\left(\mathfrak{N}_{a, b, c}\right.$, resp. $\left.\mathfrak{N}_{a+k, b, c-k}\right)$ of $\mathfrak{M}_{\chi, K^{2}}^{00}$, yet diffeomorphic through a diffeomorphism preserving the orientation and the canonical class. (For more general discussions concerning the topological, differentiable and symplectic structures we refer the reader to Freedman [16], Donaldson [13; 14], Friedman and Morgan [17], Catanese [8], Auroux and Smith [4], Gompf [18], Auroux and Katzarkov [2] and Gompf and Stipsicz [19].)

The starting point was that a partial closure $\mathfrak{N}_{a, b, c, d}$ of $\mathfrak{N}_{a, b, c, d}^{\prime}$, obtained by allowing the base to be a more general Segre-Hirzebruch surface and allowing the bidouble covers to have also du Val singularities, is, under suitable numerical conditions on $a, b, c, d$, an irreducible connected component of $\mathfrak{M}_{\chi, K^{2}}$.

Then it was proven in [12] that if $S$ is an $a b c$-surface (this means that $b=d$ ), and $S^{\prime}$ is an $a^{\prime} b^{\prime} c^{\prime}$-surface, then $S$ and $S^{\prime}$ are diffeomorphic if and only if $b=b^{\prime}, a+c=a^{\prime}+c^{\prime}$. The diffeomorphism between $S$ and $S^{\prime}$ was obtained as a consequence of a classical theorem of Kas on the diffeomorphism type of differentiable Lefschetz fibrations. Here, the holomorphic map $\tilde{\varphi}: S \rightarrow \mathbb{P}^{1}$, obtained as the composition of $\pi: S \rightarrow \mathbb{P}^{1} \times \mathbb{P}^{1}$ with the first projection, admits a small differentiable perturbation which yields a natural symplectic Lefschetz fibration $\varphi: S \rightarrow \mathbb{P}^{1}$. The bulk of the proof was to show that $\varphi, \varphi^{\prime}$ are isomorphic differentiable Lefschetz fibrations according to the criterion of Kas.

Kas [21] shows indeed that the isomorphism class of a differentiable Lefschetz fibration of genus $g \geq 2$ is completely determined by the equivalence class of its monodromy factorization in the mapping class group, for the equivalence relation generated by Hurwitz equivalence and by simultaneous conjugation.

Here, the mapping class group monodromy factorization of a Lefschetz fibration is the sequence of positive Dehn twists associated to a quasi-basis of the fundamental group $\pi_{1}\left(\mathbb{P}^{1}-\left\{b_{i}\right\}, b_{0}\right)$, whose conjugacy classes yield the local monodromies. 
The change of quasi-basis of the fundamental group $\pi_{1}\left(\mathbb{P}^{1}-\left\{b_{i}\right\}, b_{0}\right)$ leads to the Hurwitz equivalence of factorizations, while simultaneous conjugation accounts for the possible different choices of an (orientation preserving) diffeomorphism of the fibre over the base point $b_{0}$ with a standard Riemann surface of genus $g$.

On the other hand, the mapping class group monodromy factorization of this Lefschetz fibration is nothing else than the homomorphic image of the braid monodromy factorization which corresponds to the branch curve $\Delta \subset \mathbb{P}^{1} \times \mathbb{P}^{1}$ of a symplectic perturbation of $\pi$ and to its first projection onto $\mathbb{P}^{1}$, and which has factors in the braid group of the sphere $\operatorname{Br}_{n}$ (where $n$ is the vertical degree of $\Delta$ ), which is presented as follows:

$$
\operatorname{Br}_{n}=\left\{\begin{array}{l|l}
\sigma_{1}, \ldots, \sigma_{n-1} & \begin{array}{l}
\sigma_{i} \sigma_{j}=\sigma_{j} \sigma_{i}, \quad \text { if }|i-j|>1 \\
\sigma_{i} \sigma_{i+1} \sigma_{i}=\sigma_{i+1} \sigma_{i} \sigma_{i+1} \\
\sigma_{1} \cdots \sigma_{n-1} \sigma_{n-1} \cdots \sigma_{1}=1
\end{array}
\end{array}\right\}
$$

Determining whether these braid monodromy factorizations are equivalent would have been the clue to deciding about the existence of a diffeomorphism between $S, S^{\prime}$ commuting with the Lefschetz fibrations $\varphi, \varphi^{\prime}$, and yielding a canonical symplectomorphism.

Our main result says however that such a diffeomorphism cannot exist, by showing that the corresponding braid monodromy factorizations are not m-equivalent, in the terminology introduced by Auroux and Katzarkov in [2]. They used the relation of mequivalence in order to obtain invariants of symplectic 4-manifolds. The m-equivalence relation is obtained by allowing not only Hurwitz equivalence and simultaneous conjugation, but also creation/cancellation of admissible pairs of a positive and of a negative node, where a node (and then also the corresponding full twist $\beta$ ) is said to be admissible if the inverse image of the node inside the ramification divisor consists of two disjoint smooth branches.

We propose here to use the more informative name "stable equivalence" instead of m-equivalence.

With this terminology we prove the following Main Theorem:

Theorem 0.1 The braid monodromy factorizations associated to an abc-surface $S$ and to an $a^{\prime} b^{\prime} c^{\prime}$-surface $S^{\prime}$ are not stably equivalent, except in the trivial cases $a=a^{\prime}$, $b=b^{\prime}, c=c^{\prime}$ or $a=c^{\prime}, b=b^{\prime}, c=a^{\prime}$ or $a=c=b^{\prime}, a^{\prime}=c^{\prime}=b$.

The result relies on a complete description of the braid monodromy factorization class associated to $\Delta$ : this is given in Theorem 3.5, which proves indeed much more than what we need for the present purposes. 
We state here a simpler byproduct of the cited result, namely:

Theorem 0.2 There is a braid monodromy factorization of the curve $\Delta$ associated to a bidouble cover $S$ of type $a, b, c, d$ whose braid monodromy group $H \subset \mathrm{Br}_{4(b+d)}$ is generated, unless we are in the cases

$$
\text { (I) } c=2 a \text { and } d=2 b \text { or (II) } a=2 c \text { and } b=2 d
$$

by the following powers of the half-twists appearing in the figure below: $\sigma_{a_{i}}, \sigma_{c_{i}}$ for $i=1, \ldots 2 b-1, \sigma_{b_{l}}, \sigma_{d_{l}}$ for $\imath=1, \ldots, 2 d-1, \sigma_{p_{2 b}}^{2}, \sigma_{q_{2 d}}^{2}, \sigma_{s}, \sigma_{u^{\prime}}^{3}$ and $\sigma_{u^{\prime \prime}}^{3}$.
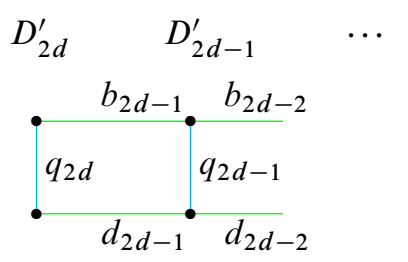

$D_{2 d}^{\prime \prime}$

$$
D_{2 d-1}^{\prime \prime}
$$

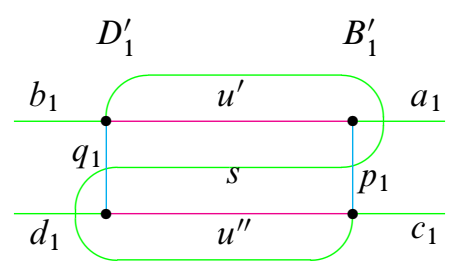

$D_{1}^{\prime \prime}$

$B_{1}^{\prime \prime}$

$\cdots$

$B_{2 b-1}^{\prime} \quad B_{2 b}^{\prime}$

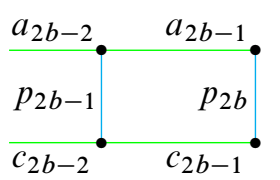

$B_{2 b-1}^{\prime \prime}$

$B_{2 b}^{\prime \prime}$

The factorization is such that

(1) each ( \pm )full-twist factor is of type $p$ or $q$,

(2) the weighted count of $( \pm)$ full-twist factors of type $p$ yields $8 a b-2(a d+b c)$,

(3) the weighted count of $( \pm)$ full-twist factors of type $q$ yields $8 c d-2(a d+b c)$.

The above result shows that the braid monodromy group $H$ depends only upon the numbers $b$ and $d$, provided for instance that we have nonvanishing of the respective numbers $8 a b-2(a d+b c), 8 c d-2(a d+b c)$, or provided that we are in the case $b=d$.

A fortiori if the groups $H$ are the same for different choices of $(a, b, c, d)$ then the fundamental groups $\pi_{1}(Q \backslash \Delta)$ are isomorphic.

This forces us to look more carefully into the problem of distinguishing classes of factorizations, and for this reason, later on, we introduce a technical novelty which consists in finding a new effective method for disproving stable-equivalence.

The method goes as follows: assume that we consider a group $G$ and a factorization of the identity in $G$

$$
\alpha_{1} \circ \cdots \circ \alpha_{m}=1
$$


and a set $\mathcal{B}$ of elements $\beta_{j} \in G$. We define stable-equivalence of factorizations with respect to $\mathcal{B}$ simply by considering the equivalence relation generated by Hurwitz equivalence, simultaneous conjugation and creation/cancellation of consecutive factors $\beta_{j} \circ \beta_{j}^{-1}$.

Then refined invariants of the stable equivalence class of the above factorization are obtained as follows:

(1) Let $H$ be the subgroup of $G$ generated by the $\alpha_{i}$ 's and let $\hat{H}$ be the subgroup of $G$ generated by the $\alpha_{i}$ 's and by the $\beta_{j}$ 's (in our case, $H$ will be called the monodromy group, and $\hat{H}$ the stabilized monodromy group).

Then $\hat{H}$ is a first invariant.

(2) Let $\mathcal{C}$ be the set of conjugacy classes $A$ in the group $H$, and let $\widehat{\mathcal{C}}$ be the set of conjugacy classes $\widehat{A}$ in the group $\hat{H}$, so that we have a natural map $\mathcal{C} \rightarrow \widehat{\mathcal{C}}$, with $A \mapsto \widehat{A}$.

Write $\widehat{\mathcal{C}}$ as a disjoint union $\hat{\mathcal{C}}^{+} \cup \hat{\mathcal{C}}^{-} \cup \widehat{\mathcal{C}}^{0}$, where $\widehat{\mathcal{C}}^{0}$ is the set of conjugacy classes of elements $a \in \widehat{H}$ which are conjugate to their inverse $a^{-1}$, and where $\hat{\mathcal{C}}^{-}$is the set of the inverse conjugacy classes of the classes in $\hat{\mathcal{C}}^{+}$.

(3) Associate to the factorization $\alpha_{1} \circ \cdots \circ \alpha_{m}=1$ the function $s: \hat{\mathcal{C}}^{+} \rightarrow \mathbb{Z}$ such that $s(c)$, for $c \in \hat{\mathcal{C}}^{+}$, is the algebraic number of occurrences of $c$ in the sequence of conjugacy classes of the $\alpha_{j}$ 's (ie, an occurrence of $c^{-1}$ counts as -1 for $s(c)$ ).

The function $s: \hat{\mathcal{C}}^{+} \rightarrow \mathbb{Z}$ is our second and most important invariant.

Remark 0.3 The calculation of the function $s: \hat{\mathcal{C}}^{+} \rightarrow \mathbb{Z}$ presupposes however a detailed knowledge of the group $\hat{H}$. For this reason it will be convenient to find some coarser derived invariant.

Substrategy I Assume that we can write $\left\{\alpha_{1}, \ldots, \alpha_{m}\right\}$ as a disjoint union $\mathcal{A}_{1} \cup \mathcal{A}_{2} \cup$ $\mathcal{D} \cup \mathcal{A}_{1}^{\prime} \cup \mathcal{A}_{2}^{\prime}$, such that the set $\mathcal{A}_{j}, j=1,2$, is contained in a conjugacy class $A_{j} \subset H$ and the set $\mathcal{A}_{j}^{\prime}, j=1,2$, is contained in the conjugacy class $A_{j}^{-1} \subset H$. Assume that the elements in $\mathcal{A}_{1} \cup \mathcal{A}_{2}$ are contained in a conjugacy class $C$ in $G$ such that $C \cap C^{-1}=\varnothing$ but that the set $\mathcal{D}$ is disjoint from the union $C \cup C^{-1}$ of the two conjugacy classes of $G$.

If we then prove that $\widehat{A_{1}} \neq \widehat{A_{2}}$ (this of course implies $A_{1} \neq A_{2}$ ) we may assume without loss of generality that $\widehat{A_{1}}, \widehat{A_{2}} \in \widehat{\mathcal{C}}^{+}$, and then the unordered pair of positive numbers $\left(\left|s\left(\widehat{A_{1}}\right)\right|, \mid s\left(\widehat{A_{2}} \mid\right)\right.$ is our derived numerical invariant of the factorization (and can easily be calculated from the cardinalities of the four sets as $\left(|| \mathcal{A}_{1}|-| \mathcal{A}_{1}^{\prime}||,|| \mathcal{A}_{2}|-| \mathcal{A}_{2}^{\prime}||\right)$. 
Substrategy II This is the strategy to show that $\widehat{A_{1}} \neq \widehat{A_{2}}$ and goes as follows. Assume further that we have a subgroup $\widetilde{H} \supset \hat{H}$ and a group homomorphism $\rho: \tilde{H} \rightarrow \Sigma$ such that $\rho(\mathcal{B})=1$. Assume also that the following key property holds.

Key property For each element $\alpha_{j} \in A_{j} \subset H$ there is an element $\widetilde{\alpha}_{j} \in \tilde{H}$ such that

$$
\alpha_{j}=\widetilde{\alpha}_{j}^{2},
$$

and moreover that this element is unique in $\widetilde{H}$ (a fortiori, it will suffice that this element is unique in $G$ ).

Proving that $\rho\left(\widetilde{\alpha}_{1}\right)$ is not conjugate to $\rho\left(\widetilde{\alpha}_{2}\right)$ under the action of $\rho(H)=\rho(\hat{H}) \subset \Sigma$ shows finally that $\alpha_{1}$ is not conjugate to $\alpha_{2}$ in $\hat{H}$, since if there is $h \in \hat{H}$ such that $\alpha_{1}=h^{-1} \alpha_{2} h$, then $\widetilde{\alpha}_{1}^{2}=\alpha_{1}=\left(h^{-1} \widetilde{\alpha}_{2} h\right)^{2}$, whence $\widetilde{\alpha}_{1}=h^{-1} \widetilde{\alpha}_{2} h$, a contradiction.

In our concrete case, we are able to determine the braid monodromy group $H$, and we observe that, since we have a so-called cuspidal factorization, all the factors $\alpha_{i}$ belong to only four conjugacy classes in the group $G$ (the classes of $\sigma_{1}, \sigma_{1}^{2}, \sigma_{1}^{-2}, \sigma_{1}^{3}$ in the braid group), corresponding geometrically to vertical tangencies of the branch curve, respectively nodes and cusps. Three of these classes are positive and only one is negative (the one of $\sigma_{1}^{-2}$ ).

We shall see that the nodes belong to conjugacy classes $A_{1}, A_{2}, A_{1}^{-1}, A_{2}^{-1} \subset H$, the positive nodes belonging to $A_{1} \cup A_{2}$, and we shall show, using a representation $\rho$ of a certain subgroup $\tilde{H}$ of "liftable" braids (ie, braids which centralize the monodromy homomorphism, whence are liftable to the mapping class group of the curve associated to the monodromy homomorphism) into a symplectic group $\Sigma$ with $\mathbb{Z} / 2$ coefficients, that the classes $\widehat{A_{1}}, \widehat{A_{2}}$ are distinct in $\hat{H}$.

Moreover, since these are positive classes in the braid group, we decree that these are classes in $\mathcal{C}^{+}$.

We are then able to easily calculate the above function for these two conjugacy classes, ie, the pair of numbers $\left(s\left(\widehat{A_{1}}\right), s\left(\widehat{A_{2}}\right)\right)$.

This new method and the above results represent the first positive step towards the realization of a more general program set up by Moishezon $[27 ; 28]$ in order to produce braid monodromy invariants which should distinguish the connected components of a moduli space $\mathfrak{M}_{\chi, K^{2}}$.

Moishezon's program is based on considering (assume here for simplicity that $K_{S}$ is ample) a general projection $\Psi_{m}: S \rightarrow \mathbb{P}^{2}$ of a pluricanonical embedding $\Phi_{m}: S \rightarrow \mathbb{P}^{P_{m}-1}$ and the braid monodromy factorization corresponding to the (cuspidal) branch curve $B_{m}$ of $\Psi_{m}$. 
An invariant of the connected component of the moduli space is then given by the equivalence class (for Hurwitz equivalence plus simultaneous conjugation) of this braid monodromy factorization. Moishezon [29], and later Moishezon and Teichner [30], calculated a coarser invariant, namely the fundamental group $\pi_{1}\left(\mathbb{P}^{2}-B_{m}\right)$.

This group turned out to be not overly complicated, and in fact, as shown in many cases by Auroux, Donaldson, Katzarkov and Yotov [1], it tends to give no extra information beyond the one given by the topological invariants of $S$ (such as $\chi, K^{2}$ ).

Auroux and Katzarkov showed that, for $m \gg 0$, the stable-equivalence class of the above braid monodromy factorization determines the canonical symplectomorphism class of $S$, and conversely.

As we have already remarked, in the case of $a b c$-surfaces the braid monodromy groups are determined by $b$ (up to conjugation), hence the fundamental groups $\pi_{1}(Q \backslash \Delta)$ are isomorphic for a fixed value of $b$.

So, Moishezon's technique produces no invariants.

There remains the problem of understanding stable-equivalence classes of pluricanonical braid monodromy factorizations: let us try to describe here how this could be done in our case.

Define $p: S \rightarrow \mathbb{P}^{2}$ as the morphism $p$ obtained as the composition of $\pi: S \rightarrow Q$ with the embedding $Q \hookrightarrow \mathbb{P}^{3}$ followed by a general projection $\mathbb{P}^{3}-\rightarrow \mathbb{P}^{2}$.

In the even more special case of $a b c$-surfaces such that $a+c=2 b$, the $m$-th pluricanonical mapping $\Phi_{m}: S \rightarrow \mathbb{P}^{P_{m}-1}$ has a (non-generic) projection given by the composition of $p$ with a Fermat type map $v_{r}: \mathbb{P}^{2} \rightarrow \mathbb{P}^{2}$ (given by $v_{r}\left(x_{0}, x_{1}, x_{2}\right)=$ $\left(x_{0}^{r}, x_{1}^{r}, x_{2}^{r}\right)$ in a suitable linear coordinate system), where $r:=m(2 b-2)$.

Let $B$ be the branch curve of a generic perturbation of $p$. Then the braid monodromy factorization corresponding to $B$ can be calculated from the braid monodromy factorization corresponding to $\Delta$.

We hope, in a sequel to this paper, to be able - relying on methods of the second author [22;23] - to determine this braid monodromy factorization: to determine whether these braid monodromy factorizations are equivalent, respectively stably-equivalent, for $a b c$-surfaces such that $a+c=2 b$ might be much harder.

The problem of calculating the braid monodromy factorization corresponding to the (cuspidal) branch curve $B_{m}$ starting from the braid monodromy factorization of $B$ has been addressed, in the special case $m=2$, by Auroux and Katzarkov [3]. Iteration of their formulae, which should in theory lead to the calculation of the braid monodromy 
factorization corresponding to the (cuspidal) branch curve $B_{m}$ (in the case, sufficient for applications, where $m$ is a sufficiently large power of 2) might however be intractable in practice.

Here are the contents of the article.

Section 1 is devoted to making precise the concept of a perturbation of $\pi$. In fact, it will be shown that a dianalytic perturbation suffices, ie, one corresponding to a covering given by equations where $\Phi, \Psi$ are either holomorphic or antiholomorphic in each single variable $x, y$.

The invariants of the branch curve $\Delta$ (genus, degree, number of nodes, cusps and vertical tangents) are computed.

Section 2 is devoted to the description of the braid monodromy group $H$ of $\Delta$, and to the conjugacy classes in $H$ of the factors of the braid monodromy factorization. The computation is based on a degeneration of the branch curves to real curves $\{f=0\}$, $\{g=0\}$ which are each the union of horizontal lines with the graph of a rational function: this type of degeneration seems to be very suitable also for writing short proofs of existing results.

Section 3 is based on the classical correspondence between 4-tuple covers and triple covers, given by the surjection $\mathfrak{S}_{4} \rightarrow \mathfrak{S}_{3}$ whose kernel is the Klein group $(\mathbb{Z} / 2)^{2}$.

We obtain a corresponding triple cover and a resulting homomorphism of its mapping class group to the symplectic group acting on the $\mathbb{Z} / 2$ homology.

This homomorphism sends the "extra" factors $\beta_{j}$ to the identity and transforms the Dehn twists $\alpha_{i}$ corresponding to the nodes of $\Delta$ to Picard Lefschetz transformations which are shown to be not conjugated under the image of the monodromy group.

\section{Perturbed simple bidouble covers}

Consider the direct sum $\mathbb{V}$ of two complex line bundles $\mathbb{L}_{1} \oplus \mathbb{L}_{2}$ on a compact complex manifold $X$, and the subset $Z$ of $\mathbb{V}$ defined by the pair of equations

$$
\begin{aligned}
z^{2} & =f(x)+w \Phi(x) \\
w^{2} & =g(x)+z \Psi(x)
\end{aligned}
$$

where $f, g$ are respective holomorphic sections of the line bundles $\mathbb{L}_{1}^{\otimes 2}, \mathbb{L}_{2}^{\otimes 2}$ (we shall also write $f \in H^{0}\left(\mathcal{O}_{X}\left(2 L_{1}\right)\right), g \in H^{0}\left(\mathcal{O}_{X}\left(2 L_{2}\right)\right)$, denoting by $L_{1}, L_{2}$ the associated Cartier divisors), and where, for the time being, $\Phi$ is a differentiable section of $\mathbb{L}_{1}^{\otimes 2} \otimes \mathbb{L}_{2}^{\otimes-1}, \Psi$ is a differentiable section of $\mathbb{L}_{2}^{\otimes 2} \otimes \mathbb{L}_{1}^{\otimes-1}$. 
Lemma 1.1 Assume that the two divisors $\{f=0\}$ and $\{g=0\}$ are smooth and intersect transversally. Then, for $|\Phi| \ll 1,|\Psi| \ll 1, Z$ is a smooth submanifold of $\mathbb{V}$, and the projection $\pi: \mathbb{V} \rightarrow X$ induces a finite covering $Z \rightarrow X$ of degree 4 , with ramification divisor (ie, critical set) $R:=\{4 z w=\Phi \Psi\}$, and with branch divisor (ie, set of critical values) $\Delta=\{\delta(x)=0\}$, where

$$
-\frac{1}{16} \delta=-f^{2} g^{2}-\frac{9}{8} f g(\Phi \Psi)^{2}+(\Psi)^{2} f^{3}+(\Phi)^{2} g^{3}+\frac{27}{16^{2}}(\Phi \Psi)^{4} .
$$

Proof $Z$ is locally defined by two complex valued functions which are holomorphic polynomials in $z$ and $w$. If we compactify the rank two vector bundle to a $\mathbb{P}_{\mathbb{C}}^{2}$-bundle, and homogenize the equations to

$$
\begin{aligned}
z^{2} & =f(x) u^{2}+w u \Phi(x) \\
w^{2} & =g(x) u^{2}+z u \Psi(x),
\end{aligned}
$$

we see that $Z$ is fibrewise the complete intersection of two degree two equations, and it does never intersect the line at infinity $\{u=0\}$, whence we get fibrewise a zero dimensional subscheme $Z_{x}$ of length 4 .

The fibre subscheme is smooth (if and only if it consists of 4 distinct points) exactly when the Jacobian determinant $4 z w-\Phi \Psi \neq 0$. This shows that outside of $R$ we have a local diffeomorphism between $Z$ and $X$.

In the case where $\Phi \equiv 0, \Psi \equiv 0, Z$ is a smooth (complex) submanifold, by the implicit function theorem, if and only if the two divisors $\{f=0\}$ and $\{g=0\}$ are smooth and intersect transversally.

If $|\Phi| \ll 1,|\Psi| \ll 1$, by uniform continuity on compact sets, the real Jacobian matrix has still rank 4 , thus $Z$ is still a real submanifold. The equation of the branch divisor $\Delta$ is obtained eliminating $z, w$ from the above three equations (see [11]).

Remark 1.2 Assume that $Z$ is a smooth submanifold. Then $R$ is smooth at the points $p \in Z$ where the fibre $Z_{x}$ has multiplicity $\mu$ at most two.

Also $\Delta$ is smooth at a point $x$ such that the fibre $Z_{x}$ consists of three distinct points.

Proof Since $p \in R$, the multiplicity $\mu \geq 2$. If equality holds, the fibre is the spectrum of $\mathbb{C}[t] /\left(t^{2}\right)$, hence by Nakayama's lemma the local ring is generated by three elements, two elements $x_{1}, x_{2}$ from $\mathcal{O}_{X}$ and an affine linear form $v(z, w)$.

It follows that $Z$ is locally biholomorphic to a hypersurface defined by $v^{2}=\varphi\left(x_{1}, x_{2}\right)$. Hence $Z$ is smooth if and only if $R=\left\{\left(x_{1}, x_{2}, v\right) \mid v=\varphi\left(x_{1}, x_{2}\right)=0\right\}$ is smooth. 
If the fibre $Z_{x}$ consists of three distinct points, then there is only one point of the fibre which is in $R$, and there the fibre has multiplicity two. Hence locally $\Delta=$ $\left\{\left(x_{1}, x_{2}\right) \mid \varphi\left(x_{1}, x_{2}\right)=0\right\}$ and therefore it is smooth.

We want to analyse now the singularities that $\Delta$ has, for a general choice of $\Phi, \Psi$. In view of the above remark, it suffices to consider points $x$ such that either

(I) there is only one point $p \in R$ above it, and with multiplicity 3 or 4 , or

(II) the fibre $R_{x}$ consists of two points with multiplicity 2 .

Definition 1.3 A point $x$ is said to be a trivial singularity of $\Delta$ if it is either a point where $f=\Phi=0$, or a point where $g=\Psi=0$.

$Z$ is said to be mildly general if

(\#) at any point where $f=\Phi=0$, we have $g \cdot \Psi \neq 0$, and symmetrically at any point where $g=\Psi=0$, we have $f \cdot \Phi \neq 0$.

Lemma 1.4 If $Z$ is mildly general, case (II) occurs exactly for the trivial singularities of $\Delta$. Moreover, case (I) occurs only for points $p \in R$ with $w(p) z(p) \neq 0$, and never leads to a point of $Z_{x}$ of multiplicity 4 .

Proof Observe that over a trivial singularity, for instance with $f=\Phi=0$, we have $z=0$, and the fibre consists of two distinct points of $R$ if $Z$ is mildly general, since $g(x) \neq 0$.

Conversely, if we have a point $p \in R_{x}$ with $z(p)=0$ and $w(p) \neq 0$, then $g(x) \neq 0$, and $\Phi \Psi(x)=0$ since $4 z w-\Phi \Psi(x)=0$.

If $\Phi(x)=0$ we have a trivial singularity, for $f=z^{2}-\Phi w$ also vanishes at $p$.

If instead $\Phi(x) \neq 0$, then the equation $f=z^{2}-\Phi w$ singles out only one value for $w$ with $z=0$, and there is no other point $p^{\prime} \in R_{x}$. In fact $p^{\prime} \neq p, p^{\prime} \in R_{x}$ implies $w\left(p^{\prime}\right)=0$, contradicting $g(x) \neq 0$ (recall that $\Psi(x)=0$ !). Thus this case cannot occur.

We argue similarly for the case $w(p)=0$ and $z(p) \neq 0$.

Claim The case where $w(p)=0=z(p)=0$ cannot occur for a point $p \in R_{x}$.

Otherwise we would have $f(x)=g(x)=\Phi \Psi(x)=0$ contradicting the hypothesis that $Z$ is mildly general.

We can finally consider the case $w(p) z(p) \neq 0$, hence $\Phi \Psi(x) \neq 0$. 
We can write our two local equations as

$$
\begin{gathered}
w=\frac{1}{\Phi(x)}\left(z^{2}-f(x)\right) \\
\left(z^{2}-f(x)\right)^{2}-z \Psi(x) \Phi(x)^{2}-g(x) \Phi(x)^{2}=0 .
\end{gathered}
$$

The form of the second equation implies that the sum of the four roots equals 0 .

Hence we conclude that not all the four roots are equal, otherwise we would have $z=0$ as fourtuple root, implying that $f(x)=\Phi(x) \Psi(x)=g(x) \Phi(x)=0$, whence we would have a trivial singularity, contradicting $w(p) z(p) \neq 0$. The last assertion is then proven.

Assume that there are two roots of multiplicity 2, thus our equation would have the form $(z-a)^{2}(z+a)^{2}$, ie $\left(z^{2}-a^{2}\right)^{2}$. Then the coefficient of $z$ would vanish, contradicting $\Phi \Psi(x) \neq 0$.

Lemma 1.5 If $Z$ is mildly general, and $|\Phi| \ll 1,|\Psi| \ll 1$, the singular points of $\Delta$ which are not the trivial singularities of $\Delta$, ie, those coming from case (I), occur only for points $p \in Z_{x}$ of multiplicity 3 which lie over arbitrarily small neighbourhoods of the points with $f(x)=g(x)=0$.

Proof By the previous lemma, we are looking for points $x$ where $\Phi \Psi(x) \neq 0$, and where the equation

$$
\left(z^{2}-f(x)\right)^{2}-z \Psi(x) \Phi(x)^{2}-g(x) \Phi(x)^{2}=0
$$

has a triple root $a$. Then the above monic equation has the form

$$
(z-a)^{3}(z+3 a)=z^{4}-6 a^{2} z^{2}+8 a^{3} z-3 a^{4} .
$$

Comparing the coefficients, we obtain

$$
\begin{aligned}
-8 a^{3} & =\Psi(x) \Phi(x)^{2} \\
f(x) & =3 a^{2} .
\end{aligned}
$$

Whence, for $|\Phi| \ll 1,|\Psi| \ll 1$, also $|a| \ll 1$, therefore also $|f(x)| \ll 1$; by symmetry we also obtain $|g(x)| \ll 1$ and our assertion is proven.

We want to describe more precisely the singularities of $\Delta$ in the case where $X$ is a complex surface. 
Proposition 1.6 Assume that $X$ is a compact complex surface, that the two divisors $\{f=0\}$ and $\{g=0\}$ are smooth and intersect transversally in a set $M$ of $m$ points. Then, for $|\Phi| \ll 1,|\Psi| \ll 1$, and for $Z$ mildly general, $Z$ is a smooth submanifold of $\mathbb{V}$, and the projection $\pi: \mathbb{V} \rightarrow X$ induces a finite covering $Z \rightarrow X$ of degree 4 , with smooth orientable ramification divisor $R:=\{4 z w=\Phi \Psi\}$, and with branch divisor $\Delta$ having as singularities precisely

(1) $3 m$ cusps lying (in triples) in an arbitrarily small neighbourhood of $M$, and moreover,

(2) the trivial singularities, which are nodes if the curve $\{f=0\}$ intersects $\{\Phi=0\}$ transversally, respectively if the curve $\{g=0\}$ intersects $\{\Psi=0\}$ transversally.

Proof Since we showed that $Z$ is smooth, by Remark 1.2 it follows that $R$ is smooth, except possibly for the points of type (I).

But in a neighbourhood of a point with $f(x)=g(x)=0, R$ is a small differentiable deformation of the nodal holomorphic curve $R_{0}$ defined ( for $\Phi(x) \equiv 0, \Psi(x) \equiv 0$ ) by the equation $4 z w=0, z^{2}=f(x), w^{2}=g(x)$.

Working locally at a point where $f(x)=g(x)=0$, we may assume that $f(x), g(x)$ are local holomorphic coordinates $\left(x_{1}, x_{2}\right)$ so that $Z$ is defined by

$$
x_{1}=z^{2}-w \Phi, \quad x_{2}=w^{2}-z \Psi,
$$

where $z, w$ are local coordinates and $\Phi, \Psi$ do not vanish.

As it was shown in [11, Section 3], in a new system of coordinates we may assume without loss of generality $\Phi \equiv \Psi \equiv \eta$, where $\eta$ is a small nonzero constant.

Thus $R$ is smooth, and it is orientable being diffeomorphic to the holomorphic curve that one gets for $\Phi(x) \equiv \eta \equiv \Psi(x)$.

We argue then as in [11] and we find that $\Delta$ has exactly three cusps as singularities for $\eta \ll 1$.

For the case (2) of the trivial singularities, it suffices to observe that the equation of $\Delta$ is given by

$$
-f^{2} g^{2}-\frac{9}{8} f g(\Phi \Psi)^{2}+(\Psi)^{2} f^{3}+(\Phi)^{2} g^{3}+\frac{27}{16^{2}}(\Phi \Psi)^{4}
$$

At a point where $f=\Phi=0$, and where $g \neq 0, \Psi \neq 0$, if we assume that $f$, $\Phi$ give a local diffeomorphism to a neighbourhood of the origin in $\mathbb{C}^{2}$, the equation becomes of order two, and with leading term $-f^{2} g^{2}(0)+(\Phi)^{2} g^{3}(0)$, thus it defines an ordinary quadratic singularity. 
Since $R$ is orientable, it follows that if the trivial singularities of $\Delta$ are nodes, then the intersection number of the two branches is \pm 1 ; the local self-intersection number is exactly equal to +1 when the two orientations of the two branches combine to yield the natural (complex) orientation of $X$.

We are going now to calculate the precise number of singularities, and of the positive, respectively negative nodes of $\Delta$ in the special case we are interested in, namely, of a perturbed bidouble cover of the quadric.

Definition 1.7 Given four integers $a, b, c, d \in \mathbb{N}_{\geq 3}$, the so-called dianalytic perturbations of simple bidouble covers are the 4-manifolds defined by equations

$$
\begin{aligned}
z^{2} & =f_{(2 a, 2 b)}\left(x_{0}, x_{1} ; y_{0}, y_{1}\right)+w \Phi_{(2 a-c, 2 b-d)}\left(x_{0}, x_{1} ; y_{0}, y_{1}\right) \\
w^{2} & =g_{(2 c, 2 d)}\left(x_{0}, x_{1} ; y_{0}, y_{1}\right)+z \Psi_{(2 c-a, 2 d-b)}\left(x_{0}, x_{1} ; y_{0}, y_{1}\right)
\end{aligned}
$$

where $f, g$ are bihomogeneous polynomials of respective bidegrees $(2 a, 2 b)$, $(2 c, 2 d)$, and where $\Phi, \Psi$ are polynomials in the ring

$$
\mathbb{C}\left[x_{0}, x_{1}, y_{0}, y_{1}, \overline{x_{0}}, \overline{x_{1}}, \overline{y_{0}}, \overline{y_{1}}\right],
$$

bihomogeneous of respective bidegrees $(2 a-c, 2 b-d),(2 c-a, 2 d-b)$ (the ring is bigraded here by setting the degree of $\overline{x_{i}}$ equal to $(-1,0)$ and the degree of $\overline{y_{i}}$ equal to $(0,-1))$.

We choose moreover $\Phi, \Psi$ to belong to the subspace where all monomials are either separately holomorphic or antiholomorphic, ie, they admit no factor of the form $x_{i} \overline{x_{j}}$ or of the form $y_{i} \overline{y_{j}}$.

Remark 1.8 Indeed we shall pick $\Phi$ (resp. $\Psi$ ) to be a product

$$
\Phi=\Phi_{1}(x) \Phi_{2}(y),
$$

where $\Phi_{1}$ is a product of linear forms, either all holomorphic or all antiholomorphic, and similarly for $\Phi_{2}$ (resp. for $\Psi_{1}, \Psi_{2}$ ).

Under this condition, at a point where $\Phi$ vanishes simply, we get

$$
\Phi=\text { unit } \cdot \Phi_{1} \quad \text { or } \quad \Phi=\text { unit } \cdot \Phi_{2} .
$$

As explained in [8, pages 134-135] to an antiholomorphic homogeneous polynomial $P\left(\overline{x_{0}}, \overline{x_{1}}\right)$ of degree $m$ we associate a differentiable section $p$ of the tensor power $\mathbb{L}^{\otimes m}$ of the tautological (negative) subbundle, such that $p(x):=P(1, \bar{x})$ inside a big disc $B(0, r)$ in the complex line having centre at the origin and radius $r$. 
We shall make the following assumptions on the polynomials $f, g, \Phi, \Psi$ :

(1) The algebraic curves $C:=\{f=0\}$ and $D:=\{g=0\}$ are smooth.

(2) $C$ and $D$ intersect transversally at a finite set $M$ contained in the product $B(0, r)^{2}$ and at these points both curves have nonvertical tangents.

(3) For both curves $C$ and $D$ the first projection on $\mathbb{P}^{1}$ is a simple covering and moreover the vertical tangents of $C$ and $D$ are all distinct.

(4) The associated perturbation is sufficiently small and mildly general.

(5) The trivial singularities are nodes with nonvertical tangents, contained in $B(0, r)^{2}$.

(6) The cusps of $\Delta$ have nonvertical tangent.

Consider the branch curve $\Delta$ of the perturbed bidouble cover. Then the defining equation $\delta$ is bihomogeneous of bidegree $(4(a+c), 4(b+d))$.

We proved that $\Delta$ has exactly $k:=12(a d+b c)$ cusps, coming from the $m:=$ $4(a d+b c)$ points of the set $M=C \cap D$.

If $\Phi, \Psi$ are holomorphic, then $\Delta$ has exactly

$$
v:=4(2 a b+2 c d-a d-b c)
$$

(positive) nodes.

In general, this number $v$ equals the difference $v^{+}-v^{-}$between the number of positive and the number of negative nodes.

In fact the nodes of $\Delta$ occur only for the trivial singularities.

A trivial singularity $f=\Phi=0$ yields a node with the same tangent cone as

$$
f^{2}-\Phi^{2} \cdot g(0)
$$

By Remark 1.8 we may assume that there are local holomorphic coordinates $f, \widehat{\Phi}$ such that either

$$
\Phi=\text { unit } \cdot \widehat{\Phi} \text { or } \Phi=\text { unit } \cdot \bar{\Phi} .
$$

In the first case we get the tangent cone of a holomorphic node, hence a positive node, in the second case we get the tangent cone of an antiholomorphic node, hence a negative node.

Lemma 1.9 The number $t_{f}$ of vertical tangents for the curve $C$ is $t_{f}=4(2 a b-a)$, while the number $t_{g}$ of vertical tangents for the curve $D$ is $t_{g}=4(2 c d-c)$. For a 
general choice of $\Phi, \Psi$, the number $t$ of vertical tangents of $\Delta$ equals $t=2 t_{f}+2 t_{g}+m$. The genus of the Riemann surface $R$ equals

$$
g=1+16(a+c)(b+d)-4(a+b+c+d)-k-v,
$$

where $k:=3 m=12(a d+b c)$ is the number of cusps of $\Delta$ and $v:=v^{+}-v^{-}$is the number of nodes of $\Delta$, counted with sign.

Proof The curve $C$ has bidegree $(2 a, 2 b)$ thus its canonical divisor has degree $2 g(C)-2=2 a(2 b-2)+2 b(2 a-2)=8 a b-4 a-4 b$. The first projection has degree $2 b$, thus by Hurwitz' formula the number of ramification points is $2 g(C)-2+4 b$. Since the morphism is simple, the number $t_{f}$ of vertical tangent equals the number of ramification points; similarly for $D$.

In the case where $\Phi \equiv 0, \Psi \equiv 0$, the ramification curve $R_{0}$ has $m$ double points, and is composed of two double covers of $C \cup D$, branched on the set of double points. Therefore, if $g$ is the genus of $R$,

$$
\begin{aligned}
2 g-2 & =2(2 g(C)-2)+2(2 g(D)-2)+4 m \\
& =16 a b-8 a-8 b+16 c d-8 c-8 d+16(a d+b c) \\
& =16(a+c)(b+d)-8(a+b+c+d) .
\end{aligned}
$$

Now, the first projection yields a map $p: R \rightarrow \mathbb{P}_{\mathbb{C}}^{1}$ of degree $4(b+d)$, thus the formula for $t$ is again an application of Hurwitz' formula since $\left.p\right|_{R}$ is finite (the map from $\Delta$ to $\mathbb{P}_{\mathbb{C}}^{1}$ being finite, for $\Phi, \Psi$ sufficiently small).

The surface $R$ is smooth orientable, and $p$ has only a finite number of critical values. Thus $p$ is oriented, and indeed a ramified covering. For general $\Phi, \Psi$, it has simple ramification only, with distinct critical values. Hence the number of critical values is given by

$$
\begin{aligned}
2 g-2+8(b+d) & =16(a+c)(b+d)-8(a+c) \\
& =2 t_{f}+2 t_{g}+16(a d+b c) \\
& =2 t_{f}+2 t_{g}+4 m \\
& =2 t_{f}+2 t_{g}+m+k .
\end{aligned}
$$

We conclude observing that the number of critical values equals the number $k$ of (nonvertical) cusps plus the number $t$ of vertical tangents.

Remark 1.10 (1) Observe that the fact that $p: R \rightarrow \mathbb{P}_{\mathbb{C}}^{1}$ has a finite number of critical points implies immediately that $p$ is finite, since there is then a finite set in $\mathbb{P}_{\mathbb{C}}^{1}$ such that over its complement we have a covering space. Thus $p$ is orientation preserving and each noncritical point contributes positively to the degree of the map $p$. 
(2) Consider now a real analytic 1-parameter family $Z(\eta)$ such that the perturbation terms $\Phi_{\eta}, \Psi_{\eta} \rightarrow 0$. Then we have a family of ramification points $P_{i}(\eta)$ which tend to the ramification points of $p_{0}: R_{0} \rightarrow \mathbb{P}_{\mathbb{C}}^{1}$. For $R_{0}$, a double cover of $C \cup D$, we have $2 t_{f}$ ramification points lying over the $t_{f}$ vertical tangents of $C, 2 t_{g}$ ramification points lying over the $t_{g}$ vertical tangents of $D$, whereas the $m$ nodes of $R_{0}$ are limits of 4 ramification points, three cusps and a simple vertical tangent. This is the geometric reason why $t=2 t_{f}+2 t_{g}+m$; it also tells us where to look for the vertical tangents of $\Delta$.

In the next section we shall also consider the curves $C$ and $D$ as small real deformations of reducible real curves with only nodes (with real tangents) as singularities: according to the classical notation, these will be called improper nodes.

Moreover, we shall also consider real polynomials $\Phi, \Psi$, such that the trivial singularities are also given by real points. By the results of [11], from the perturbation of each proper node of $C \cup D$, ie, of a point of $M=C \cap D$, we shall obtain a real vertical tangent, a real cusp, and two imaginary cusps.

\section{The basic model}

In this section we will provide a model with some choices of $f, g, \Phi, \Psi$ to which the reasoning of the previous chapter applies. In particular they are explicit enough to collect a lot of geometric information and to determine the braid monodromy factorization in the sequel.

Let us begin with real polynomials $f^{\times}$and $g^{\times}$defining nodal curves $C^{\times}$, respectively $D^{\times}$.

For the sake of simplicity we replace here bihomogeneous polynomials $f\left(x_{0}, x_{1} ; y_{0}, y_{1}\right)$ by their restrictions to the affine open set $x_{0}=1, y_{0}=1$ and write $f(x, y)$ for $f(1, x ; 1, y)$.

We let $f^{\times}:=f_{1} \cdots f_{2 b}$ defining $f_{i}:=y-2 i$ for $i=2, \ldots, 2 b$ but setting

$$
f_{1}:=(y-2) \prod_{i=1}^{2 a}(x-2 i)+\prod_{i=1}^{2 a-1}(x-2 i-1),
$$

and we define similarly $g^{\times}:=g_{1} \cdots g_{2 d}$ setting $g_{i}=y+2 i$ for $\mathrm{i}=2, \ldots, 2 d$ except that we set

$$
g_{1}:=(y+2) \prod_{i=1}^{2 c}(x+2 i)-\prod_{i=1}^{2 c-1}(x+2 i+1) .
$$

See Figure 1 for an example. 


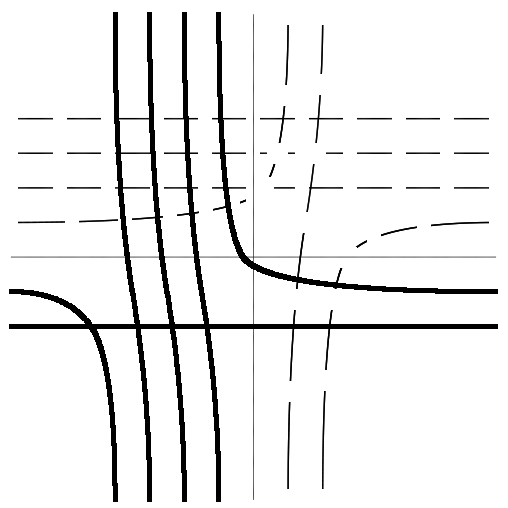

Figure 1: Real part of $C^{\times}$(bold) and $D^{\times}$(dashed) in case $(a, b, c, d)=(1,2,2,1)$

Remark 2.1 Note that the equations of $f_{1}$ and $g_{1}$ are chosen in such a way that their zero sets are graphs of rational functions $\widetilde{f}_{1}$, respectively $\widetilde{g}_{1}$, of $x$ which, regarded as maps from $\mathbb{C}$ to $\mathbb{C}$, preserve the real line. Moreover, $\widetilde{f}_{1}$ preserves both the upper and lower halfplane, while $\widetilde{g}_{1}$ exchanges them.

In fact, $\tilde{f}_{1}$ has no critical points on $\mathbb{P}_{\mathbb{R}}^{1}$ and

$$
\operatorname{deg}\left(\left.\tilde{f}_{1}\right|_{\mathbb{P}_{\mathbb{R}}^{1}}\right): \mathbb{P}_{\mathbb{R}}^{1} \rightarrow \mathbb{P}_{\mathbb{R}}^{1}=2 a=\operatorname{deg}\left(\tilde{f}_{1}\right),
$$

and similarly for $\tilde{g}_{1}$.

Next we define a real analytic 1-parameter family of dianalytic perturbation terms $\Phi_{\eta}$ and $\Psi_{\eta}$ introducing the following notation:

$$
\begin{aligned}
\Phi_{(2 a-c, 0)} & = \begin{cases}\prod_{j=1}^{2 a-c}(x-4 a-2 j) & \text { if } 2 a \geq c, \\
\prod_{j=1}^{c-2 a}(\bar{x}-4 a-2 j) & \text { if } 2 a<c .\end{cases} \\
\Phi_{(0,2 b-d)} & = \begin{cases}\prod_{j=1}^{2 b-d}(y-4 b-2 j) & \text { if } 2 b \geq d, \\
\prod_{j=1}^{d-2 b}(\bar{y}-4 b-2 j) & \text { if } 2 b<d .\end{cases}
\end{aligned}
$$




$$
\begin{aligned}
& \Psi_{(2 c-a, 0)}= \begin{cases}\prod_{j=1}^{2 c-a}(x+4 c+2 j) & \text { if } 2 c \geq a, \\
\prod_{j=1}^{a-2 c}(\bar{x}+4 c+2 j) & \text { if } 2 c<a .\end{cases} \\
& \Psi_{(0,2 d-b)}= \begin{cases}\prod_{j=1}^{2 d-b}(y+4 d+2 j) & \text { if } 2 d \geq b, \\
\prod_{j=1}^{b-2 d}(\bar{x}+4 d+2 j) & \text { if } 2 d<b .\end{cases} \\
& \Phi_{\eta}:=\eta \Phi_{(2 a-c, 2 b-d)}=\eta \Phi_{(2 a-c, 0)} \Phi_{(0,2 b-d)} . \\
& \Psi_{\eta}:=\eta \Psi_{(2 c-a, 2 d-b)}=\eta \Psi_{(2 c-a, 0)} \Psi_{(0,2 d-b)} .
\end{aligned}
$$

See Figure 2 for an illustration.

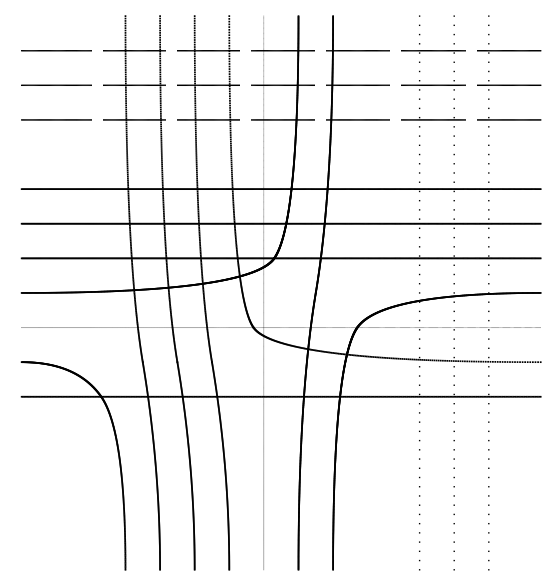

Figure 2: Zero sets of $\Phi$ (dashed) and $\Psi$ (dotted) added

Given $f^{\times}$, consider the polynomial $f: f^{\times}+c_{f}$, where $c_{f}$ is a small constant; likewise consider $g: g^{\times}+c_{g}$. Adding these small constants to the respective equations of $C^{\times}$ and $D^{\times}$we get polynomials $f$ and $g$ which define smooth curves $C$ and $D$. We have more precisely:

Proposition 2.2 If the constants $c_{f}, c_{g}, \eta$ are chosen sufficiently small, the polynomials $f, g, \Phi, \Psi$ thus obtained satisfy the following list of hypotheses (cf page 365) for $r>8(a+b+c+d)$ : 
(1) The algebraic curves $C=\{f=0\}$ and $D=\{g=0\}$ are smooth.

(2) $C$ and $D$ intersect transversally at a finite set $M$ contained in the bidisc $B(0, r)^{2}$ and at these points both curves have nonvertical tangents.

(3) For both curves $C$ and $D$ the first projection on $\mathbb{P}^{1}$ is a simple covering and moreover the vertical tangents of $C$ and $D$ are all distinct.

(4) The associated perturbation is mildly general.

(5) The trivial singularities of $\Delta$ are nodes with nonvertical tangents, contained in $B(0, r)^{2}$.

(6) The cusps of $\Delta$ have nonvertical tangent.

Proof Property (1) follows obviously from Bertini's theorem, since we have pencils without base points.

Property (2) follows since the conditions are open conditions which hold for the nodal curve $C^{\times}$and $D^{\times}$, as we shall show in Lemma 2.3 below.

By the same lemma the critical values for $C^{\times} \cup D^{\times}$are distinct, hence property (3) is proven if we show that the two vertical tangents arising from each improper node do not map to the same critical value. If this were the case, the local degree of the projection would be 4 and not 2 .

To prove property (4) that the associated perturbation is mildly general, it suffices - by definition and continuity - to note that no three of the functions $f^{\times}, g^{\times}, \Phi, \Psi$ have a common zero.

With Proposition 1.6 we deduce from (4) that the trivial singularities are nodes, which yields the first part of (5). Moreover the local analysis in the proof of Proposition 1.6 shows that the two tangents at the node are close to the tangent to $C \cup D$ at that point. So the second part of (5) follows by continuity, since the points of $C^{\times} \cup D^{\times}$with vertical tangents are not on the zero sets of $\Phi$ and $\Psi$.

For (6), as shown in [11, Section 3], and already used in Proposition 1.6, we may assume that $f, g$ are local coordinates $U, V$, and that $\Phi, \Psi$ are small constants $\eta_{1}, \eta_{2}$. So the local equation of $\Delta$ is

$$
-U^{2} V^{2}-\frac{9}{8} U V c^{2}+c^{2} V^{3}+U^{3}+\frac{27}{16^{2}} c^{4},
$$

where $c$ is the ratio $\eta_{1} / \eta_{2}=\Phi / \Psi$.

Following the proof in [11, Section 3], we set $c=\lambda^{3}$, and see that setting $U=\lambda^{4} u_{0}$, $V=\lambda^{4} v_{0}$ the equation becomes

$$
-u_{0}^{2} v_{0}^{2}-\frac{9}{8} u_{0} v_{0} c^{2}+c^{2} v_{0}^{3}+u_{0}^{3}+\frac{27}{16^{2}}
$$


Then the directions of the tangent at the three cusps in the coordinates $(U, V)$ are nonconstant functions of $c$.

Hence for a general choice of the ratio $c$ between $\Phi(0)$ and $\Psi(0)$ these tangents are not vertical.

Lemma 2.3 The critical values of the vertical projection of $C^{\times} \cup D^{\times}$are all distinct and real. Moreover,

(*) $C^{\times}$and $D^{\times}$intersect transversally at a finite set $M^{\times}$contained in the bidisc $B(0, r)^{2}$ and at these points both curves have nonvertical tangents

Proof Each critical point is a singular point of the union $C^{\times} \cup D^{\times}$since the components of these curves are horizontal sections. Given two components of $C^{\times} \cup D^{\times}$ we can easily check that the number of real points in their intersection equals the intersection number of these two components. Hence all singularities are real, they are nodes, and moreover all critical values are real.

Recall now that each component of $C^{\times}$except $\left\{f_{1}=0\right\}$ is a horizontal line, and similarly for $D^{\times}$we have only the component $\left\{g_{1}=0\right\}$ which is not a horizontal line. Hence the nodes are of three types: type $C$, where $\left\{f_{1}=0\right\}$ meets a horizontal component of $D^{\times}$, mixed type, where $f_{1}=g_{1}=0$, and type $D$, where $g_{1}=0$ meets a horizontal component of $C^{\times}$.

A vertical line through a point of mixed type cannot contain points of type $C$, nor $D$, so we have to exclude that a vertical line contains a point of type $C$ and a point of type $D$. But we simply observe that a point of type $C$ has positive abscissa, while a point of type $D$ has negative abscissa.

To prove $(*)$ we note again that all singularities are nodes and we check from the equations that their coordinates are bounded by $8(a+b+c+d)$. Since all components of $C^{\times} \cup D^{\times}$map biregularly to the base, no component has a vertical tangent.

We introduce now a geometric system of paths in the $\mathbb{C}$-line with coordinate $x$ (this is the part not at infinity of the target space $\mathbb{P}^{1}$ of the vertical projection):

$$
\gamma_{v, \rho}, \quad(v, \rho) \in \mathcal{V}=\mathcal{V}^{+} \cup \mathcal{V}^{-}
$$

where $\quad \mathcal{V}^{+}=\left\{(v, \rho) \mid 1 \leq v \leq 4 a,\left\{\begin{array}{ll}1<\rho \leq 2 b & \text { if } v \text { odd } \\ -2 d \leq \rho \leq-1 & \text { if } v \text { even }\end{array}\right\}\right.$,

respectively, $\mathcal{V}^{-}=\left\{(v, \rho) \mid 1 \leq-v \leq 4 c,\left\{\begin{array}{ll}1 \leq \rho \leq 2 b & \text { if } v \text { odd } \\ -2 d \leq \rho<-1 & \text { if } v \text { even }\end{array}\right\}\right.$, 
correspond to the singular points of $C^{\times} \cup D^{\times}: \mathcal{V}^{+}$corresponds to those with positive abscissa, $\mathcal{V}^{-}$corresponds instead to those with negative abscissa.

Observe in fact that the number of positive critical values is $2 a(2 b+2 d-1)$ and thus equals the cardinality of $\mathcal{V}^{+}$.

The set $\mathcal{V}^{+}$, respectively $\mathcal{V}^{-}$, is ordered lexicographically according to $|\nu|$ and $\rho$, and these orderings correspond each to the total order of the critical values on the real line given by the absolute value.

The total order of $\mathcal{V}$ is then determined by requiring that elements in $\mathcal{V}^{+}$precede those in $\mathcal{V}^{-}$.

The $\gamma_{\nu, \rho}$ 's are a system of simple paths based at the origin, following each other in counterclockwise order, each $\gamma_{\nu, \rho}$ going around (again in a counterclockwise way) the corresponding critical value.

In fact we can choose the paths with $v$ positive to lie in the upper half plane except in a neighbourhood of the critical value (lying on the positive real line) which they encircle. Similarly the paths with $v$ negative can be chosen to lie in the lower half plane except in a neighbourhood of the critical value (lying on the negative real line) which they encircle. See Figure 3.

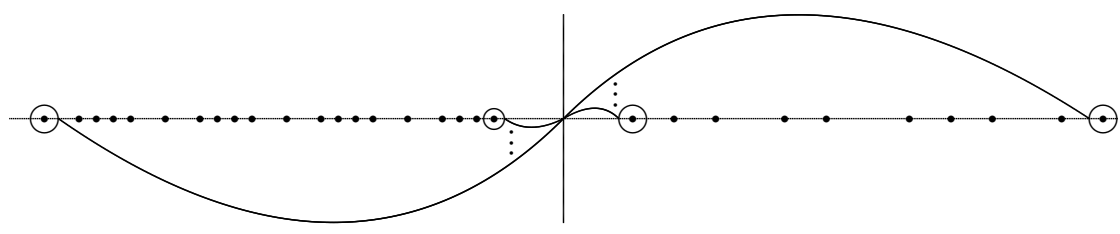

Figure 3: The geometric system for the curve of Figure 1

To explain the choice of the index set in more detail let us observe some restrictions for the $y$-coordinate function on the different components of $C^{\times} \cup D^{\times}$when the $x$-coordinate is restricted to be real and positive: it is constant of value $2 i$, resp. $-2 j$ for the components defined by $f_{i}$ resp. $g_{j}$ with $i, j>1$ and bounded between -1 and -2 for the component defined by $g_{1}$. It is real or infinity for the component $C_{1}$ defined by $f_{1}$ and is monotone increasing outside of the poles.

Since the $y$-coordinate on $C_{1}$ takes value in $[2,3]$ for $x=0$, we can precisely describe in which order the other components are intersected by $C_{1}$ as $x$ increases.

First $C_{1}$ intersects the other components $C_{2}, \ldots, C_{2 b}$ of $C^{\times}$in this order at points labelled by $(1,2), \ldots,(1,2 b)$; then the $x$-coordinate crosses a pole of $f_{1}$ and $y$ changes sign. 
Next $C_{1}$ intersects the components $D_{2 d}, \ldots, D_{1}$ of $D^{\times}$in this order at points labelled by $(2,-2 d), \ldots,(2,-1)$.

After that the $y$ coordinate changes sign again, and the same pattern of intersections repeats itself over and over till the $y$-coordinate finally tends to 2 as the $x$-coordinate tends to infinity.

We can finally summarize how our choice of the index set relates to this sequence of intersections:

- a critical value with $v>0$ corresponds to an intersection with $C_{1}$,

- each time the $y$-coordinate changes sign, $v$ is incremented by one,

- a critical value with $\rho$ positive corresponds to an intersection with the component defined by $f_{\rho}$,

- a critical value with $\rho$ negative corresponds to an intersection with the component defined by $g_{-} \rho$.

Of course the situation over the negative real line can be described similarly and we have:

- a critical value with $v$ negative corresponds to an intersection point with the component defined by $g_{1}$,

- each time the $y$-coordinate changes sign over a negative real $x$, the absolute value of the index $v$ is incremented by one.

In the reference fibre over the origin the roots of $f^{\times}$are real and positive, in fact the root of $f_{i}$ is $2 i$ for $i>1$ and between 2 and 3 for $i=1$. Similarly the roots of $g^{\times}$ are real and negative, the root of $g_{j}$ is $-2 j$ except for $g_{1}$ when the root is between -2 and -1 .

We define now arcs in the reference fibre, contained in the upper half-plane and connecting two roots according to the following labelling:

$$
\tau_{i, j}: \begin{cases}i=1, j>1 & \text { between roots of } f_{1}, f_{j} \\ i>0, j<0 & \text { between roots of } f_{i}, g_{|j|} \\ i<0, j=-1 & \text { between roots of } g_{1}, g_{|i|}\end{cases}
$$

and subject to the following rule: for each fixed $i$ (resp. $j$ ) the arcs $\tau_{i, j}$ follow each other in counterclockwise order without intersecting outside of the end points, and otherwise two such arcs intersect transversally and in at most one point.

These arcs are thus determined up to an isotopy fixing a neighbourhood of infinity. 
Proposition 2.4 The braid monodromy factorization for the curve $C^{\times} \cup D^{\times}$with respect to the geometric basis $\gamma_{\nu, \rho}$ can be given in terms of the mapping classes $\beta_{i, j}$, the half-twists associated to the arcs $\tau_{i, j}$, in the following form:

$$
\gamma_{\nu, \rho} \mapsto \begin{cases}\beta_{1, \rho}^{2} & \text { if } v>0, \\ \beta_{\rho,-1}^{2} & \text { if } v<0 .\end{cases}
$$

Proof Since all critical points on $C^{\times} \cup D^{\times}$for the vertical projection are nodes, the mapping class associated to any element $\gamma_{v, \rho}$ of the geometric system is necessarily a full twist on some arc between two roots.

These two roots are in fact the endpoints of $\tau_{1, \rho}$, resp. $\tau_{\rho,-1}-$ depending on the sign of $v$ - due to the way the index pair $(v, \rho)$ remembers the two components which intersect at the critical value encircled by $\gamma_{\nu, \rho}$.

It thus remains to prove that the mapping class associated to $\gamma_{\nu, \rho}$ can be obtained from an arc in the upper half plane.

Let us consider the case $v>0$. Then we specify the geometric loop $\gamma_{v, \rho}$ as follows: a simple arc in the upper half plane, which will be called the "tail", connecting the base point with a point on the real line, which we shall call the "fork point", followed by a circle around the critical value corresponding to $(\nu, \rho)$ run once counterclockwise before the loop goes back along the tail.

When $\gamma_{v, \rho}$ runs the circle around the critical value we get a mapping class in the fibre over the fork point of $\gamma_{v, \rho}$ which is obviously the full twist on the straight real arc connecting the roots associated to the two components of $C^{\times} \cup D^{\times}$which intersect at the critical point.

Along the tail of $\gamma_{v, \rho}$ all roots stay fixed for the horizontal components, the root associated to $g_{1}=0$ moves in the lower half plane, the root associated to $f_{1}=0$ moves in the upper half plane, cf Remark 2.1.

We may thus associate continuously to each point $x$ on the tail of $\gamma_{\nu, \rho}$ an arc $\tau_{x}$ in the fibre $F_{x}$ over $x$, such that the mapping class associated to $\gamma_{\nu, \rho}$ truncated at $x$ is given by the full twist on $\tau_{x}$.

In fact this arc can be chosen to be the straight arc except for $x$ on $\gamma_{v, \rho}$ close to the origin, because for $x=0$ we would get the straight arc through other roots.

By continuity also the arc associated to $x=0$ may be chosen to lie in the upper half plane and it is thus isotopic to $\tau_{1, \rho}$, as we wanted to show.

The argument in the case $v<0$ is the same. 
We have now reached a point where we can draw the first two consequences concerning the braid monodromy homomorphism associated to the vertical projection of the branch curve $\Delta$.

By continuity we may deduce first the following claim about the mapping classes associated to the divisor $\Delta$ along the paths $\gamma_{v, \rho}$. To state it properly let $A_{i, j}$ be respective closed neighbourhoods of the arcs $\tau_{i, j}$, each containing, among the roots of $f^{\times} g^{\times}$, only the two roots which form the endpoints of $\tau_{i, j}$.

Proposition 2.5 Let $\Delta$ be defined as in Proposition 2.2 and choose sufficiently small constants so that it moreover lies in a sufficiently close neighbourhood of $C^{\times} \cup D^{\times}$. Then the following holds true:

(1) Each $\gamma_{v, \rho}$ encircles all critical values of $p_{1}: \Delta \rightarrow \mathbb{P}^{1}$ which tend to the corresponding critical value of $C^{\times} \cup D^{\times}$.

(2) The mapping class associated to $\gamma_{\nu, \rho}$ is supported on $A_{i, j}$ if $\beta_{i, j}^{2}$ is the mapping class associated in the case of the nodal curve $C^{\times} \cup D^{\times}$.

Of course we will henceforth tacitly assume to be in the situation of the above proposition.

The other consequence is that all mapping classes associated to the paths $\gamma_{\nu, \rho}$ are given by braids lying in a distinguished subgroup of the braid group. To define this subgroup consider the 2-cable homomorphism $\mathrm{Br}_{n}$ to $\mathrm{Br}_{2 n}$, which associates to each braid a corresponding braid with doubled strands, for example, as in the figure:

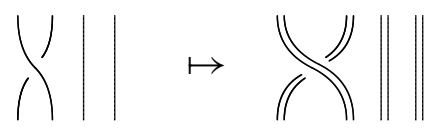

\subsection{Definition}

Define the 2-cable homomorphism for each braid $P_{i}(t)(i=1, \ldots, n)$ as the braid $Q_{j}(t)(j=1, \ldots, 2 n)$ with $Q_{2 i}(t):=P_{i}(t), Q_{2 i+1}(t):=P_{i}(t)+\epsilon$, for $\epsilon \ll 1$, so that $4 \epsilon<\min _{t \in[0,1], i \neq j}\left|P_{i}(t)-P_{j}(t)\right|$.

Define the subgroup $\mathrm{CBr}_{n} \subset \mathrm{Br}_{2 n}$ of 2- cable braids to be the subgroup generated by the image of the 2-cable homomorphism, and by the half-twists inside each cable (ie, a standard half twist involving $Q_{2 i}(0), Q_{2 i+1}(0)$, which remain at distance $\epsilon$ ). 
The half-twists inside the cables generate a free abelian normal subgroup of rank $n$, thus leading, via the 2-cable homomorphism, to a representation of $\mathrm{CBr}_{n}$ as the semidirect product

$$
\mathrm{CBr}_{n} \cong \mathbb{Z}^{n} \rtimes \mathrm{Br}_{n} .
$$

Here $\mathbb{Z}^{n}:=\left\langle\sigma_{1+2 i}\right\rangle_{i=0, \ldots, n-1}$ and $\mathrm{Br}_{n}:=\left\langle\sigma_{j}^{\prime}\right\rangle_{j=1, \ldots, n-1}$, where

$$
\sigma_{j}^{\prime}:=\sigma_{2 j} \sigma_{2 j-1} \sigma_{2 j+1} \sigma_{2 j} .
$$

Lemma 2.6 The braid monodromy associated to $\Delta$ maps the homotopy class of any path $\gamma_{v, \rho}$ to the subgroup $\mathrm{CBr}_{n}$ of $\mathrm{Br}_{2 n}$.

Moreover the braid monodromy associated to $C^{\times} \cup D^{\times}$along $\gamma_{v, \rho}$ is the image of the above through the natural projection $\mathrm{CBr}_{n} \rightarrow \mathrm{Br}_{n}$.

(Note that $n$ now is the vertical degree of $C^{\times} \cup D^{\times}$, while the vertical degree of $\Delta$ is $2 n$.)

Proof Braids in $\mathrm{CBr}_{n}$ can be realized as braided ribbons, where each doubled strand is considered as the boundary of a twisted ribbon. Along any path $\gamma_{\nu, \rho}$ the braid associated to $\Delta$ can be extended to a ribbon: we simply connect each pair of roots regenerating from a single point of $C \cup D$ by a straight arc and observe that such arcs never intersect, since the roots of $C$ and $D$ stay sufficiently away from each other along $\gamma_{v, \rho}$.

The second assertion is obvious.

In the next step we investigate the local braid monodromy factorizations in more detail for the regenerations of proper and improper nodes of $C^{\times} \cup D^{\times}$.

In the case of a proper node we get a cusp-cluster, four critical points of which three are cusps and the last is a vertical tangency point. In the case of an improper node we get a cluster of vertical tangents, four critical points, all of which are vertical tangency points.

The braid monodromy for the regeneration into a cusp-cluster has been thoroughly investigated before in [11], where the braid monodromy factorization type has been determined up to Hurwitz equivalence and simultaneous conjugation. ${ }^{2}$

We take this investigation over again in order to determine the Hurwitz equivalence class of the factorization from the datum of the product of the factors.

\footnotetext{
${ }^{2}$ The factorization was explicitly described for a particular choice of coordinates $(x, y)$. However, any other coordinate change, $\hat{x}=F(x), \hat{y}=G(x, y)$, induces another description of the fibre bundle induced by $p(x, y):=x$ on the complement of $\Delta$. This results into a Hurwitz equivalence (action of the base diffeomorphism $F$ ) followed by a simultaneous conjugation (action of the diffeomorphism $G$ on the reference fibre).
} 
Proposition 2.7 The braid monodromy factorization of a (regenerated) cusp-cluster with product $\sigma_{2}^{3} \sigma_{1} \sigma_{3} \sigma_{2} \sigma_{1}^{2} \sigma_{3}^{2}$ is Hurwitz-equivalent to the factorization

$$
\sigma_{2}^{3} \circ \sigma_{1} \sigma_{3} \sigma_{2} \sigma_{3}^{-1} \sigma_{1}^{-1} \circ \sigma_{1}^{3} \circ \sigma_{3}^{3} \text {. }
$$

Proof By the results of [11] the two factorizations are equivalent up to Hurwitzequivalence and simultaneous conjugation. Since these two operations commute the braid monodromy factorization of our cusp-cluster is Hurwitz equivalent to a factorization

$$
\left(\sigma_{2}^{3}\right)^{\gamma} \circ\left(\sigma_{1} \sigma_{3} \sigma_{2} \sigma_{3}^{-1} \sigma_{1}^{-1}\right)^{\gamma} \circ\left(\sigma_{1}^{3}\right)^{\gamma} \circ\left(\sigma_{3}^{3}\right)^{\gamma}
$$

where, as usual, $\beta^{\gamma}:=\gamma \beta \gamma^{-1}$.

Since Hurwitz equivalence does not change the product of a factorization, it follows that $\gamma$ is in the centralizer of the braid $\sigma_{2}^{3} \sigma_{1} \sigma_{3} \sigma_{2} \sigma_{1}^{2} \sigma_{3}^{2}$.

From the work of Gonzales-Meneses and Wiest [20] we may read off two elements, $\Delta_{4}:=\sigma_{1} \sigma_{2} \sigma_{3} \sigma_{1} \sigma_{2} \sigma_{1}$ and $\sigma_{1}^{-1} \sigma_{2} \sigma_{1}$, which generate this centralizer.

To prove our claim it thus suffices to give a Hurwitz equivalence between the factorization given in the above formula and its conjugates by $\sigma_{1}^{-1} \sigma_{2} \sigma_{1}$, respectively $\Delta_{4}$. Below we first give expressions for the two respective conjugated factorizations using the fact that $\sigma_{i}^{\Delta_{4}}=\sigma_{4-i}$.

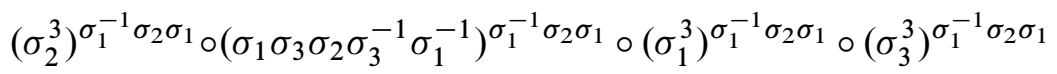

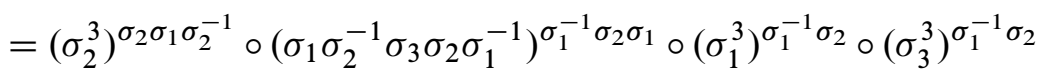

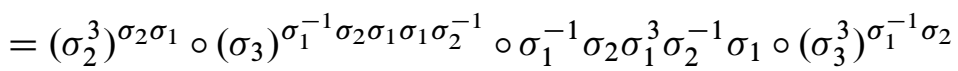

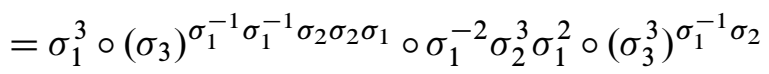

$$
\begin{aligned}
& =\sigma_{1}^{3} \circ\left(\sigma_{3}\right)^{\sigma_{1}^{-2} \sigma_{2}^{2}} \circ\left(\sigma_{2}^{3}\right)^{\sigma_{1}^{-2}} \circ\left(\sigma_{2}^{3}\right)^{\sigma_{1}^{-1} \sigma_{3}^{-1}} \\
& \left(\sigma_{2}^{3}\right)^{\Delta_{4}} \circ\left(\sigma_{1} \sigma_{3} \sigma_{2} \sigma_{3}^{-1} \sigma_{1}^{-1}\right)^{\Delta_{4}} \circ\left(\sigma_{1}^{3}\right)^{\Delta_{4}} \circ\left(\sigma_{3}^{3}\right)^{\Delta_{4}}=\sigma_{2}^{3} \circ \sigma_{3} \sigma_{1} \sigma_{2} \sigma_{1}^{-1} \sigma_{3}^{-1} \circ \sigma_{3}^{3} \circ \sigma_{1}^{3}
\end{aligned}
$$

Finally we show that these two elements are in fact in the same Hurwitz-equivalence class by performing elementary Hurwitz operations and rewriting of the factors. Here the notation $\sim_{(i+1)}$ is employed to denote a simple Hurwitz move affecting the positions $i$ and $i+1$.

$$
\begin{gathered}
\sigma_{2}^{3} \circ \sigma_{1} \sigma_{3} \sigma_{2} \sigma_{3}^{-1} \sigma_{1}^{-1} \circ \sigma_{1}^{3} \circ \sigma_{3}^{3} \\
\sim_{(23)} \sigma_{2}^{3} \circ \sigma_{1}^{3} \circ\left(\sigma_{1} \sigma_{3} \sigma_{2} \sigma_{3}^{-1} \sigma_{1}^{-1}\right)^{\sigma_{1}^{-3}} \circ \sigma_{3}^{3} \\
=\sigma_{2}^{3} \circ \sigma_{1}^{3} \circ \sigma_{1}^{-2} \sigma_{3} \sigma_{2} \sigma_{3}^{-1} \sigma_{1}^{2} \circ \sigma_{3}^{3}
\end{gathered}
$$




$$
\begin{aligned}
& \sim_{(12)} \sigma_{1}^{3} \circ\left(\sigma_{2}^{3}\right)^{\sigma_{1}^{-3}} \circ \sigma_{1}^{-2} \sigma_{3} \sigma_{2} \sigma_{3}^{-1} \sigma_{1}^{2} \circ \sigma_{3}^{3}
\end{aligned}
$$

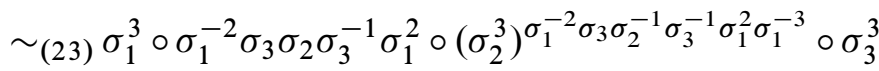

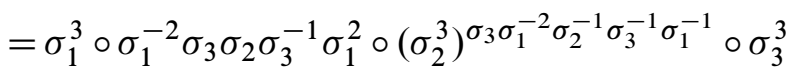

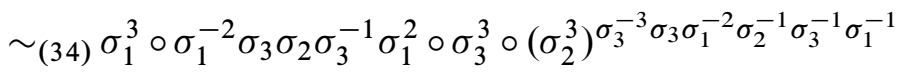

$$
\begin{aligned}
& =\sigma_{1}^{3} \circ \sigma_{1}^{-2} \sigma_{3} \sigma_{2} \sigma_{3}^{-1} \sigma_{1}^{2} \circ \sigma_{3}^{3} \circ\left(\sigma_{1}^{3}\right)^{\sigma_{3}^{-2} \sigma_{1}^{-2} \sigma_{2}^{-1} \sigma_{3}^{-1} \sigma_{2}}
\end{aligned}
$$

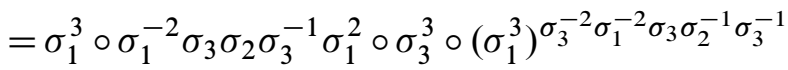

$$
\begin{aligned}
& =\sigma_{1}^{3} \circ \sigma_{1}^{-2} \sigma_{3} \sigma_{2} \sigma_{3}^{-1} \sigma_{1}^{2} \circ \sigma_{3}^{3} \circ\left(\sigma_{1}^{3}\right)^{\sigma_{3}^{-1} \sigma_{1}^{-1} \sigma_{1}^{-1} \sigma_{2}^{-1}} \\
& =\sigma_{1}^{3} \circ \sigma_{1}^{-2} \sigma_{3} \sigma_{2} \sigma_{3}^{-1} \sigma_{1}^{2} \circ \sigma_{3}^{3} \circ\left(\sigma_{2}^{3}\right)^{\sigma_{1}^{-1} \sigma_{3}^{-1}} \\
& =\sigma_{1}^{3} \circ \sigma_{1}^{-2} \sigma_{3} \sigma_{2} \sigma_{3}^{-1} \sigma_{1}^{2} \circ \sigma_{3}^{3} \circ\left(\sigma_{3}^{3}\right)^{\sigma_{1}^{-1} \sigma_{2}}
\end{aligned}
$$

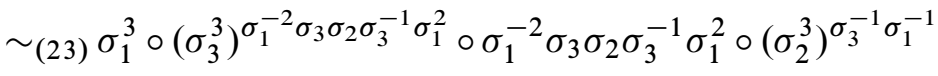

$$
\begin{aligned}
& =\sigma_{1}^{3} \circ\left(\sigma_{3}^{3}\right)^{\sigma_{1}^{-2} \sigma_{3} \sigma_{2}} \circ \sigma_{1}^{-2} \sigma_{3} \sigma_{2} \sigma_{3}^{-1} \sigma_{1}^{2} \circ\left(\sigma_{2}^{3}\right)^{\sigma_{3}^{-1} \sigma_{1}^{-1}} \\
& =\sigma_{1}^{3} \circ\left(\sigma_{2}^{3}\right)^{\sigma_{1}^{-2}} \circ \sigma_{1}^{-2} \sigma_{3} \sigma_{2} \sigma_{3}^{-1} \sigma_{1}^{2} \circ\left(\sigma_{2}^{3}\right)^{\sigma_{3}^{-1} \sigma_{1}^{-1}} \\
& =\sigma_{1}^{3} \circ \sigma_{1}^{-2} \sigma_{2}^{3} \sigma_{1}^{2} \circ\left(\sigma_{2}\right)^{\sigma_{1}^{-2} \sigma_{3}} \circ\left(\sigma_{2}^{3}\right)^{\sigma_{3}^{-1} \sigma_{1}^{-1}}
\end{aligned}
$$

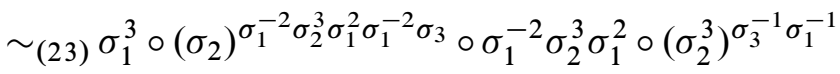

$$
\begin{aligned}
& =\sigma_{1}^{3} \circ\left(\sigma_{2}\right)^{\sigma_{1}^{-2} \sigma_{2}^{2} \sigma_{2} \sigma_{3}} \circ\left(\sigma_{2}^{3}\right)^{\sigma_{1}^{-2}} \circ\left(\sigma_{2}^{3}\right)^{\sigma_{3}^{-1} \sigma_{1}^{-1}} \\
& =\sigma_{1}^{3} \circ\left(\sigma_{3}\right)^{\sigma_{1}^{-2} \sigma_{2}^{2}} \circ\left(\sigma_{2}^{3}\right)^{\sigma_{1}^{-2}} \circ\left(\sigma_{2}^{3}\right)^{\sigma_{1}^{-1} \sigma_{3}^{-1}} \\
& \sigma_{2}^{3} \circ \sigma_{1} \sigma_{3} \sigma_{2} \sigma_{3}^{-1} \sigma_{1}^{-1} \circ \sigma_{1}^{3} \circ \sigma_{3}^{3} \\
& \sim_{(34)} \sigma_{2}^{3} \circ \sigma_{1} \sigma_{3} \sigma_{2} \sigma_{3}^{-1} \sigma_{1}^{-1} \circ \sigma_{3}^{3} \circ \sigma_{1}^{3}
\end{aligned}
$$

Comparison now shows that we got the same elements.

In the case of a regeneration into a cluster of vertical tangents we have to understand first the braid monodromy factorization type up to Hurwitz equivalence and simultaneous conjugation.

Proposition 2.8 The braid monodromy factorization associated to a cluster of tangents corresponding to an improper node is given by

$$
\sigma_{2} \sigma_{3} \sigma_{2}^{-1} \circ \sigma_{1} \sigma_{2} \sigma_{1}^{-1} \circ \sigma_{2} \sigma_{3} \sigma_{2}^{-1} \circ \sigma_{1} \sigma_{2} \sigma_{1}^{-1}
$$

up to Hurwitz equivalence and simultaneous conjugation. 
Proof Without loss of generality, we assume that we have an improper node of $C^{\times}$.

We may take a coordinate change not altering the $x$ coordinate such that the local equation of $C^{\times}$is $u(x, y)\left(y^{2}-x^{2}\right)=0$, where $u(x, y) \neq 0$ (we also have $g^{\times}(x, y) \neq 0$ ).

Consider first a small perturbation with $\Psi=0$ : then the branch curve is given by

$$
f^{\times 2} g^{\times 2}-g^{\times 3} \Phi^{2}=g^{\times 2}\left(f^{\times 2}-g^{\times} \Phi^{2}\right)=0 .
$$

The vertical tangents can be located from the nonunit factor.

$$
\begin{aligned}
\frac{\partial}{\partial y}\left(f^{\times 2}-g^{\times} \Phi^{2}\right)=0 & \Longleftrightarrow \frac{\partial}{\partial y}\left(\left(y^{2}-x^{2}\right)^{2}-g^{\times} u^{-2} \Phi^{2}\right)=0 \\
& \Longleftrightarrow 2 y\left(y^{2}-x^{2}\right)=\frac{\partial}{\partial y}\left(g^{\times} u^{-2} \Phi^{2}\right)
\end{aligned}
$$

To simplify the equations we take a local model based on an apt choice of $\Phi$ with $\left(g^{\times} u^{-2} \Phi^{2}\right) \equiv \epsilon^{2}$, where $\epsilon$ is a small constant, which makes the right hand side vanish. The two equations have solutions $y=0$ and $x^{4}=\epsilon^{2}$, which leads to four solutions close to $(0,0)$.

Then it is not hard to see that the same braid monodromy factorization is obtained from the model

$$
\left(y^{2}-x^{2}-\epsilon\right)\left(y^{2}-x^{2}+\epsilon\right)=0
$$

for which the factorization is as claimed.

The actual perturbation $\Delta$ of $C^{\times} \cup D^{\times}$is linked to the above through a path of perturbations which constantly have four vertical tangency points at the cluster. So the local fibration does not change and therefore also the equivalence class of braid monodromy factorization for Hurwitz equivalence and simultaneous conjugation stays the same.

Proposition 2.9 Suppose that a braid monodromy factorization of a cluster of tangents is Hurwitz plus conjugation equivalent to the factorization given by

$$
\left(\sigma_{1}^{-1} \sigma_{2} \sigma_{1}\right) \circ\left(\sigma_{2}^{-1} \sigma_{3} \sigma_{2}\right) \circ\left(\sigma_{1}^{-1} \sigma_{2} \sigma_{1}\right) \circ\left(\sigma_{2}^{-1} \sigma_{3} \sigma_{2}\right) .
$$

Then the two factorizations are equivalent via Hurwitz equivalence and simultaneous conjugation by a power of $\sigma_{1}$ if and only if their products are equal.

Proof Again we deduce immediately that their products are equal if and only if the first factorization is Hurwitz equivalent to the given one via conjugation by some braid centralizing the product. 
The product is however $\left(\Delta_{4} \sigma_{1}^{-2} \sigma_{3}^{-2}\right)^{2}$ and by [20] its centralizer is generated by $\Delta_{4}$ and $\sigma_{1}$.

This immediately shows that the given equivalence preserves the product.

In the other direction, in order to prove our claim it thus suffices to give a Hurwitz equivalence between the factorization of the claim and its conjugate by $\Delta_{4}$.

We first give the expression for this conjugate factorization using that $\Delta_{4} \sigma_{i} \Delta_{4}^{-1}=\sigma_{4-i}$.

$$
\begin{aligned}
& \left(\sigma_{1}^{-1} \sigma_{2} \sigma_{1}\right)^{\Delta_{4}} \circ\left(\sigma_{2}^{-1} \sigma_{3} \sigma_{2}\right)^{\Delta_{4}} \circ\left(\sigma_{1}^{-1} \sigma_{2} \sigma_{1}\right)^{\Delta_{4}} \circ\left(\sigma_{2}^{-1} \sigma_{3} \sigma_{2}\right)^{\Delta_{4}} \\
= & \left(\sigma_{3}^{-1} \sigma_{2} \sigma_{3}\right) \circ\left(\sigma_{2}^{-1} \sigma_{1} \sigma_{2}\right) \circ\left(\sigma_{3}^{-1} \sigma_{2} \sigma_{3}\right) \circ\left(\sigma_{2}^{-1} \sigma_{1} \sigma_{2}\right)
\end{aligned}
$$

We finish providing an elementary chain of transformations, obtained using only Hurwitz equivalence, simultaneous conjugation by $\sigma_{1}^{2}$ and rewriting of factors, which leads from the factorization of the claim to its conjugate by $\Delta_{4}$.

$$
\begin{aligned}
& \left(\sigma_{1}^{-1} \sigma_{2} \sigma_{1}\right)^{\sigma_{1}^{2}} \circ\left(\sigma_{2}^{-1} \sigma_{3} \sigma_{2}\right)^{\sigma_{1}^{2}} \circ\left(\sigma_{1}^{-1} \sigma_{2} \sigma_{1}\right)^{\sigma_{1}^{2}} \circ\left(\sigma_{2}^{-1} \sigma_{3} \sigma_{2}\right)^{\sigma_{1}^{2}} \\
& =\left(\sigma_{1} \sigma_{2} \sigma_{1}^{-1}\right) \circ\left(\sigma_{2}^{-1} \sigma_{3} \sigma_{2}\right)^{\sigma_{1}^{2}} \circ\left(\sigma_{1}^{-1} \sigma_{2} \sigma_{1}\right)^{\sigma_{1}^{2}} \circ\left(\sigma_{2}^{-1} \sigma_{3} \sigma_{2}\right)^{\sigma_{1}^{2}}
\end{aligned}
$$

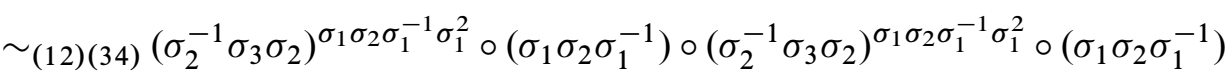

$$
\begin{aligned}
& =\left(\sigma_{2}^{-1} \sigma_{3} \sigma_{2}\right)^{\sigma_{1} \sigma_{2} \sigma_{1}} \circ\left(\sigma_{1} \sigma_{2} \sigma_{1}^{-1}\right) \circ\left(\sigma_{2}^{-1} \sigma_{3} \sigma_{2}\right)^{\sigma_{1} \sigma_{2} \sigma_{1}} \circ\left(\sigma_{1} \sigma_{2} \sigma_{1}^{-1}\right) \\
& =\left(\sigma_{2}^{-1} \sigma_{3} \sigma_{2}\right)^{\sigma_{2} \sigma_{1} \sigma_{2}} \circ\left(\sigma_{1} \sigma_{2} \sigma_{1}^{-1}\right) \circ\left(\sigma_{2}^{-1} \sigma_{3} \sigma_{2}\right)^{\sigma_{2} \sigma_{1} \sigma_{2}} \circ\left(\sigma_{1} \sigma_{2} \sigma_{1}^{-1}\right) \\
& =\left(\sigma_{3}\right)^{\sigma_{2} \sigma_{1}} \circ\left(\sigma_{1} \sigma_{2} \sigma_{1}^{-1}\right) \circ\left(\sigma_{3}\right)^{\sigma_{2} \sigma_{1}} \circ\left(\sigma_{1} \sigma_{2} \sigma_{1}^{-1}\right) \\
& =\left(\sigma_{3}\right)^{\sigma_{2}} \circ\left(\sigma_{1} \sigma_{2} \sigma_{1}^{-1}\right) \circ\left(\sigma_{3}\right)^{\sigma_{2}} \circ\left(\sigma_{1} \sigma_{2} \sigma_{1}^{-1}\right) \\
& =\left(\sigma_{2} \sigma_{3} \sigma_{2}^{-1}\right) \circ\left(\sigma_{1} \sigma_{2} \sigma_{1}^{-1}\right) \circ\left(\sigma_{2} \sigma_{3} \sigma_{2}^{-1}\right) \circ\left(\sigma_{1} \sigma_{2} \sigma_{1}^{-1}\right) \\
& =\left(\sigma_{3}^{-1} \sigma_{2} \sigma_{3}\right) \circ\left(\sigma_{2}^{-1} \sigma_{1} \sigma_{2}\right) \circ\left(\sigma_{3}^{-1} \sigma_{2} \sigma_{3}\right) \circ\left(\sigma_{2}^{-1} \sigma_{1} \sigma_{2}\right)
\end{aligned}
$$

Corollary 2.10 In the situation of the above proposition the two factorizations are equivalent up to Hurwitz equivalence and creation/deletion of admissible pairs.

Proof It suffices to show that simultaneous conjugation by $\sigma_{1}^{2}$ can be induced by Hurwitz moves and creation/deletion of admissible pairs. This can be done as follows:

$$
\begin{aligned}
& \sigma_{1}^{-1} \sigma_{2} \sigma_{1} \circ \sigma_{2}^{-1} \sigma_{3} \sigma_{2} \circ \sigma_{1}^{-1} \sigma_{2} \sigma_{1} \circ \sigma_{2}^{-1} \sigma_{3} \sigma_{2} \\
& \sim_{c} \sigma_{1}^{-2} \circ \sigma_{1}^{2} \circ \sigma_{1}^{-1} \sigma_{2} \sigma_{1} \circ \sigma_{2}^{-1} \sigma_{3} \sigma_{2} \circ \sigma_{1}^{-1} \sigma_{2} \sigma_{1} \circ \sigma_{2}^{-1} \sigma_{3} \sigma_{2} \\
& \sim \sigma_{1}^{-2} \circ\left(\sigma_{1}^{-1} \sigma_{2} \sigma_{1}\right)^{\sigma_{1}^{2}} \circ\left(\sigma_{2}^{-1} \sigma_{3} \sigma_{2}\right)^{\sigma_{1}^{2}} \circ\left(\sigma_{1}^{-1} \sigma_{2} \sigma_{1}\right)^{\sigma_{1}^{2}} \circ\left(\sigma_{2}^{-1} \sigma_{3} \sigma_{2}\right)^{\sigma_{1}^{2}} \circ \sigma_{1}^{2} \\
& \sim \sigma_{1}^{-2} \circ \sigma_{1}^{2} \circ\left(\sigma_{1}^{-1} \sigma_{2} \sigma_{1}\right)^{\sigma_{1}^{2}} \circ\left(\sigma_{2}^{-1} \sigma_{3} \sigma_{2}\right)^{\sigma_{1}^{2}} \circ\left(\sigma_{1}^{-1} \sigma_{2} \sigma_{1}\right)^{\sigma_{1}^{2}} \circ\left(\sigma_{2}^{-1} \sigma_{3} \sigma_{2}\right)^{\sigma_{1}^{2}}
\end{aligned}
$$

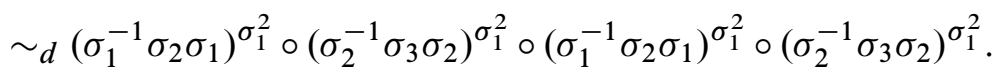


A braid monodromy factorization is associated to the branch curve $\Delta$ once we fix a geometric system of paths for the critical values of $\Delta$ in the base and a system of arcs between the roots in the reference fibre $F_{0}$.

Concerning the geometric system of paths, we shall construct them later as a "regeneration" of the old system of paths $\gamma_{\nu, \rho}$ : ie given a path $\gamma_{\nu, \rho}$ we shall determine a sequence of consecutive paths - actually four of them - in the new system, having the same tail, and such that their product is homotopic to $\gamma_{v, \rho}$. In particular, the choice for these paths will be a local one around the critical values for $C^{\times} \cup D^{\times}$.

The choice of the local factorization of each $\gamma_{v, \rho}$ corresponds to a choice inside the Hurwitz equivalence class of the braid factorization whose product is the braid associated to $\gamma_{\nu, \rho}$.

We shall now concentrate on the systems of arcs.

\section{Proof of the factorization theorem}

Proposition 3.1 Up to homotopy in small discs containing pairs $D_{i}^{\prime}, D_{i}^{\prime \prime}$ or $B_{J}^{\prime}, B_{J}^{\prime \prime}$ the punctured fibre $F_{0}$ is given in the following picture:

\begin{tabular}{|c|c|c|c|c|c|}
\hline$D_{2 d}^{\prime}$ & $D_{2 d-1}^{\prime}$ & $\cdots$ & $D_{1}^{\prime}$ & $B_{1}^{\prime}$ & $B_{2 b-1}^{\prime}$ \\
\hline • & • & & • & • & • \\
\hline • & • & & • & • & • \\
\hline$D_{2 d}^{\prime \prime}$ & $D_{2 d-1}^{\prime \prime}$ & $\cdots$ & $D_{1}^{\prime \prime}$ & $B_{1}^{\prime \prime}$ & $B_{2 b-1}^{\prime \prime}$ \\
\hline
\end{tabular}

The monodromy $\theta$ of the perturbed bidouble cover, with respect to the origin, is given by

$$
\begin{aligned}
\omega_{D_{j}^{\prime}} & \mapsto(12), & \omega_{B_{j}^{\prime}} & \mapsto(13), \\
\omega_{D_{j}^{\prime \prime}} & \mapsto(34), & \omega_{B_{j}^{\prime \prime}} & \mapsto(24),
\end{aligned}
$$

where $\omega_{P}$ is any simple closed path around $P$ not crossing the imaginary axis $i \mathbb{R} \subset F_{0}$.

Proof As we remarked before, in the unperturbed Galois cover case we have real points $D_{i}, B_{j}$, only, the $D_{i}$ 's with positive real coordinate, the $B_{j}$ 's with negative real coordinate. 
And the monodromy is (12)(34), resp. (13)(24) for paths which do not cross the imaginary axis.

The deformation then splits each branch point into two, hence the corresponding monodromies (which are transpositions) must be (12) and (34), resp. (13) and (24) (which commute). After a suitable homotopy the points are in the positions given by the picture.

Referring to the above figure we introduce the following notation for a system of arcs, which are uniquely determined (up to homotopy) by their endpoints and the property that they are monotonous in the real coordinate and do not pass below any puncture; ie, if they share the real coordinate with a puncture they have larger imaginary coordinate.

$$
\begin{array}{ll}
p_{i}: B_{i}^{\prime}, B_{i}^{\prime \prime} & q_{\imath}: D_{l}^{\prime}, D_{l}^{\prime \prime} \\
a_{i j}: B_{i}^{\prime}, B_{j}^{\prime} & b_{l J}: D_{l}^{\prime}, D_{J}^{\prime} \\
c_{i j}: B_{i}^{\prime \prime}, B_{j}^{\prime \prime} & d_{l J}: D_{l}^{\prime \prime}, D_{J}^{\prime \prime} \\
u_{i_{J}}^{\prime}: B_{i}^{\prime}, D_{J}^{\prime} & u_{i J}^{\prime \prime}: B_{i}^{\prime \prime}, D_{J}^{\prime \prime}
\end{array}
$$

(See Figure 4 below representing $u_{1 j}^{\prime}$ and $u_{1 j}^{\prime \prime}$.)

Remark 3.2 We can safely assume that any such arc is fully contained in one of the previously defined closed sets $A_{i, j}$ (neighbourhood of the arc $\tau_{i, j}$ ).

Proposition 3.3 The braid monodromy factorization associated to $\Delta$ and to a subsystem of paths refining $\gamma_{v, \rho}$ is given by

$$
\begin{array}{ll}
\sigma_{a_{1 i}} \circ \sigma_{p_{1}}^{2} \sigma_{c_{1 i}} \sigma_{p_{1}}^{-2} \circ \sigma_{a_{1 i}} \circ \sigma_{p_{1}}^{2} \sigma_{c_{1 i}} \sigma_{p_{1}}^{-2}, & \text { if } v>0 \text { and } i=\rho>0, \\
\sigma_{b_{1 j}} \circ \sigma_{q_{1}}^{2} \sigma_{d_{1 j}} \sigma_{q_{1}}^{-2} \circ \sigma_{b_{1 j}} \circ \sigma_{q_{1}}^{2} \sigma_{d_{1 j}} \sigma_{q_{1}}^{-2}, & \text { if } v<0 \text { and } j=-\rho>0,
\end{array}
$$

up to Hurwitz equivalence and simultaneous conjugation by some power of $\sigma_{p_{1}}^{2}$ in case (1), resp. by $\sigma_{q_{1}}^{2}$ in case (2).

Proof The proofs of both cases are the same modulo an appropriate exchange of indices, so we consider the first case only.

Since the paths refine $\gamma_{\nu, \rho}$ the associated mapping classes are supported on $A_{1, i}$ for $i=\rho$, by (3) of Proposition 2.5.

Hence by Proposition 2.8 the factorization coincides with the factorization of the claim up to simultaneous conjugation by a mapping class (of this disc) supported on $A_{1, i}$. The products (ie, the product of the factorization (1), and the product of the factorization 
we are looking for, yielding the braid monodromy associated to $\gamma_{\nu, \rho}$ ) are therefore equal up to that conjugation.

To proceed we have to get more information about the second product: being associated to the path $\gamma_{v, \rho}$ it belongs to the subgroup $\mathrm{CBr}_{n}$ of $\mathrm{Br}_{2 n}$ by Lemma 2.6.

Next, the product belongs to the subgroup of braids which are supported on $A_{1, i}$. This subgroup is generated by $\sigma_{a_{1 i}}, \sigma_{c_{1 i}}, \sigma_{p_{1}}, \sigma_{p_{i}}$ and we can easily determine a presentation of its intersection with $\mathrm{CBr}_{n}$ :

$\left\langle\sigma_{p_{1}}, \sigma_{p_{i}}, \Delta_{4}:=\sigma_{a_{1 i}} \sigma_{p_{1}}^{2} \sigma_{c_{1 i}} \sigma_{p_{i}}^{2} \mid \sigma_{p_{1}} \sigma_{p_{i}}=\sigma_{p_{i}} \sigma_{p_{1}}, \Delta_{4} \sigma_{p_{1}}=\sigma_{p_{i}} \Delta_{4}, \Delta_{4} \sigma_{p_{i}}=\sigma_{p_{1}} \Delta_{4}\right\rangle$

Again by Lemma 2.6 we know the image of the product under the quotient map $\mathrm{CBr}_{n} \rightarrow \mathrm{Br}_{n}$ to be a full twist. Since both $\sigma_{p_{1}}$ and $\sigma_{p_{i}}$ belong to the kernel of that map we deduce now that this product can be written as $\Delta_{4}^{2} \sigma_{p_{1}}^{k} \sigma_{p_{i}}^{k^{\prime}}$ with $k+k^{\prime}=-8$ to match the total degree, which is 4 .

The final fact to be exploited is that the two products are conjugate inside the group generated by $\sigma_{a_{1 i}}, \sigma_{c_{1 i}}, \sigma_{p_{1}}, \sigma_{p_{i}}$.

The first product can be shown to be equal to $\Delta_{4}^{2} \sigma_{p_{1}}^{-4} \sigma_{p_{i}}{ }^{-4}$ since the two elements $\sigma_{p_{1}}$ and $\sigma_{p_{i}}$ commute, while conjugation by $\Delta_{4}$ exchanges them. Since moreover the element $\Delta_{4}^{2}$ is central we get conjugation equivalences

$$
\Delta_{4}^{2} \sigma_{p_{1}}^{k} \sigma_{p_{i}}^{k^{\prime}} \sim \Delta_{4}^{2} \sigma_{p_{1}}^{-4} \sigma_{p_{i}}{ }^{-4} \Longrightarrow \sigma_{p_{1}}^{k} \sigma_{p_{i}}^{k^{\prime}} \sim \sigma_{p_{1}}^{-4} \sigma_{p_{i}}{ }^{-4}
$$

But closing the braid on both sides we get a four component link for the right hand side, which consists of two unlinked copies of the $(4,2)$ torus link.

This soon implies that, since the braids are conjugate, $k$ and $k^{\prime}$ are both even, hence the left hand side consists of the unlinked union of a $(k, 2)$ torus link with a $\left(k^{\prime}, 2\right)$ torus link.

The nonzero linking numbers on the right are equal to 2 , while the nonzero linking numbers on the left are equal to $|k| / 2,\left|k^{\prime}\right| / 2$.

Again since the braids are conjugate and the linking numbers are invariant under conjugation we conclude from $|k|=\left|k^{\prime}\right|=2$ and $k+k^{\prime}=-4$ that $k=k^{\prime}=-2$, so both products are equal.

So finally we may invoke Proposition 2.9 and we are left only with the ambiguity of a conjugation by a power of $\sigma_{p_{1}}$.

This power must however have an even exponent, since the group of liftable braids is left invariant by conjugation by $\sigma_{p_{1}}^{2}$, while conjugation by $\sigma_{p_{1}}$ carries $\sigma_{a_{1 i}}$ outside of the subgroup of liftable braids. 


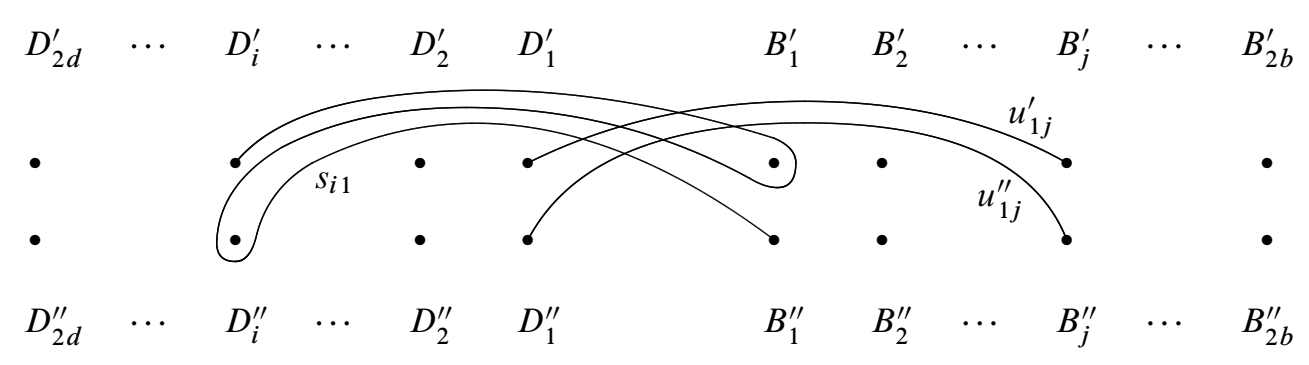

Figure 4: Representatives of certain arcs of type $u^{\prime}, u^{\prime \prime}$ and $s$

To state our next claim we need to introduce some further arcs $s_{i j}$ contained the corresponding discs $A_{i j}$. They are determined by fact that the relative position of $s_{i j}$ to $u_{i_{j}}^{\prime}$ and $u_{i_{j}}^{\prime \prime}$ (cf Figure 4) is the same as the relative position of $s$ to $u^{\prime}$ and $u^{\prime \prime}$ in Figure 5 below.

Proposition 3.4 Suppose $v$ and $\rho$ have opposite sign. Then the braid monodromy factorization associated to $\Delta$ and to a subsystem of paths refining $\gamma_{\nu, \rho}$ is given by

$$
\sigma_{u_{i j}}^{3} \circ \sigma_{s_{i j}} \circ \sigma_{u_{i_{j}}^{\prime}}^{3} \circ \sigma_{u_{i_{j}}^{\prime \prime}}^{3} \quad \text { where } \begin{cases}i=1, j=-\rho & \text { if } v>0 \\ i=\rho, j=1 & \text { if } v<0\end{cases}
$$

up to Hurwitz equivalence. In particular there is a distinguished refining subsystem of paths such that the associated factorization is precisely the one given above.

Proof Since the paths refine $\gamma_{\nu, \rho}$, by Proposition 2.5 (3) the factors are supported on $A_{i, j}$, which is a topological disc containing four roots, $B_{i}^{\prime}, B_{i}^{\prime \prime}, D_{j}^{\prime}, D_{j}^{\prime \prime}$. Locally the divisor $\Delta$ is a regeneration of a node between the two components $C$ and $D$, thus by the results of Catanese and Wajnryb [11] the factorization coincides with the factorization of the claim up to Hurwitz equivalence and simultaneous conjugation. Hence also their products coincide up to conjugation.

These products are both contained in $\mathrm{CBr}_{n}$, cf Lemma 2.6, and map to the same braid $\beta_{i, j}^{2}$ under the quotient map $\mathrm{CBr}_{n} \rightarrow \mathrm{Br}_{n}$. Hence they coincide up to conjugation by elements in the subgroup generated by the half-twists on the arcs $p_{i}$ and $q_{j}$. Since these half-twists are central in $\mathrm{CBr}_{n}$, the products do in fact coincide.

Therefore we may invoke Proposition 2.7 to deduce that the factorization is Hurwitz equivalent to the one given in the claim.

In particular the Hurwitz action on the factors is induced by the Hurwitz action on the subsystem of paths, thus we may pick the subsystem of paths in the Hurwitz orbit which maps to the given factorization. 
Theorem 3.5 The factorization is a product of factorizations

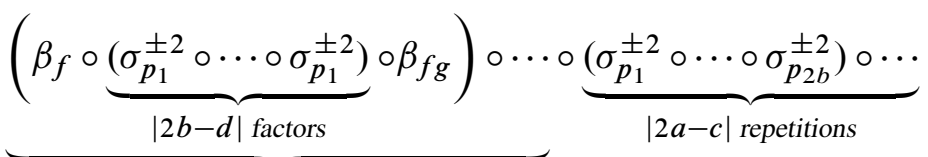

$2 a$ repetitions

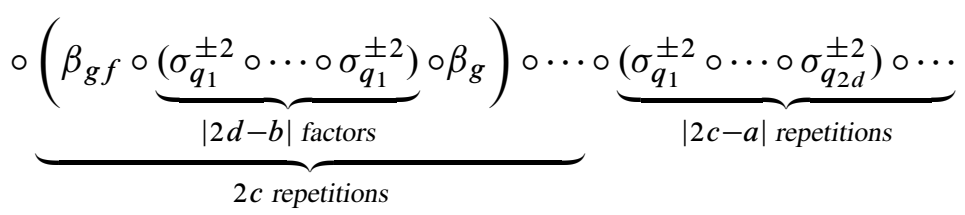

where the sign of the exponents 2 is constant inside a pair of brackets and is the sign of the number which determines the number of factors, ie $(2 b-d)$ resp. $(2 a-c),(2 c-a)$ or $(2 d-b)$, and where the $\beta$ 's further decompose as products of factorizations

$$
\begin{aligned}
& \beta_{f}=\beta_{f, 2} \circ \cdots \circ \beta_{f, 2 b}, \quad \beta_{f g}=\beta_{f g, 2 d} \circ \cdots \circ \beta_{f g, 1}, \\
& \beta_{g}=\beta_{g, 2} \circ \cdots \circ \beta_{g, 2 d}, \quad \beta_{g f}=\beta_{g f, 2 b} \circ \cdots \circ \beta_{g f, 1},
\end{aligned}
$$

based on elementary factorizations each having four factors

$$
\begin{aligned}
& \beta_{f, i}=\sigma_{a_{1 i}} \circ \sigma_{p_{1}}^{2} \sigma_{c_{1 i}} \sigma_{p_{1}}^{-2} \circ \sigma_{a_{1 i}} \circ \sigma_{p_{1}}^{2} \sigma_{c_{1 i}} \sigma_{p_{1}}^{-2}, \quad \beta_{f g, j}=\sigma_{u_{1 j}}^{3} \circ \sigma_{s_{1 j}} \circ \sigma_{u_{1 j}^{\prime}}^{3} \circ \sigma_{u_{1 j}^{\prime \prime}}^{3}, \\
& \beta_{g, j}=\sigma_{b_{1 j}} \circ \sigma_{q_{1}}^{2} \sigma_{d_{1 j}} \sigma_{q_{1}}^{-2} \circ \sigma_{b_{1 j}} \circ \sigma_{q_{1}}^{2} \sigma_{d_{1 j}} \sigma_{q_{1}}^{-2}, \quad \beta_{g f, i}=\sigma_{u_{i 1}}^{3} \circ \sigma_{s_{i 1}} \circ \sigma_{u_{i 1}^{\prime}}^{3} \circ \sigma_{u_{i 1}^{\prime \prime}}^{3}
\end{aligned}
$$

The elementary factorizations originate in the regeneration of nodes of the branch curve of the corresponding bidouble Galois-cover.

Proof Consider first the set of critical values for the vertical projection of $\Delta$. They are either arbitrarily close to the critical values of the vertical projection of $C^{\times} \cup D^{\times}$ or they are images of the nodes of $\Delta$ and thus are real.

We choose an associated system of paths in the base such that

(1) the paths associated to positive critical values of nodes belong to the upper half plane,

(2) the paths associated to negative critical values of nodes belong to the lower half plane,

(3) the paths associated to the four critical values which regenerate from a node of $C^{\times} \cup D^{\times}$refine the path $\gamma_{v, \rho}$ associated to the value of that node.

We have still the choices of elements in the Hurwitz orbits of subsystems refining each path $\gamma_{\nu, \rho}$. We use these choices to adjust the system of paths using Proposition 3.3 and Proposition 3.4 and we succeed to get the elementary factorizations, at least up to the conjugations mentioned in Proposition 3.3. 
The mapping classes associated to the other paths are obviously full twists. We have to handle the problem that there may be critical values corresponding to different critical points: this happens if and only if there are nodes coming from vertical components of $\Phi, \Psi$.

We can handle this difficulty adding a constant to $\Phi$, resp. $\Psi$. This resolves the problem except for the case that the divisor is not ample, ie, if $2 b=d$ or $2 b=d$.

In this case we take a local differentiable perturbation at the nodes.

We pass now to consider the conjugacy classes of the braids appearing in the braid monodromy factorization, in order to prove Theorem 0.2.

Lemma 3.6 All full-twists of type $p$ and $q$ are admissible.

Proof The arc of type $p_{i}$ is homotopic to the union of two paths $\gamma_{D_{i}^{\prime}}, \gamma_{D_{i}^{\prime \prime}}$ in the positive half plane. By Proposition 3.1 we see that the monodromies around the two ends are disjoint transpositions, hence the full-twist on $p_{i}$ is admissible. The same argument yields the claim for $q_{J}$.

\subsection{Generators of mapping class groups}

Lemma 3.7 If $a_{i j}, a_{j k}, a_{i k}$ are the sides of a topological triangle taken in positive order, then

$$
\sigma_{a_{i j}} \sigma_{a_{j k}}=\sigma_{a_{j k}} \sigma_{a_{i k}}
$$

Proof This is easily seen from the identity $\sigma_{1} \sigma_{2}=\sigma_{2}\left(\sigma_{2}^{-1} \sigma_{1} \sigma_{2}\right)$ in the braid group $\mathrm{Br}_{3}$ which maps homomorphically onto the group generated by $\sigma_{a_{i j}}, \sigma_{a_{j k}}, \sigma_{a_{i k}}$ via

$$
\sigma_{1} \mapsto \sigma_{a_{i j}}, \quad \sigma_{2} \mapsto \sigma_{a_{j k}}, \quad \sigma_{2}^{-1} \sigma_{1} \sigma_{2} \mapsto \sigma_{a_{i k}}
$$

Lemma 3.8 The following subgroups of the braid group $\mathrm{Br}_{4(b+d)}$ are identical:

$$
\left\langle\sigma_{a_{1,2}}, \sigma_{a_{2,3}}, \ldots, \sigma_{a_{n-1, n}}\right\rangle, \quad\left\langle\sigma_{a_{1,2}}, \ldots, \sigma_{a_{1, n}}\right\rangle, \quad\left\langle\sigma_{a_{i j}}, 1 \leq i, j \leq n\right\rangle,
$$

Proof By the relation in Lemma 3.7 a group containing $\sigma_{a_{i j}}, \sigma_{a_{j k}}$ also contains $\sigma_{a_{i k}}$ for any triple $i, j, k$. Hence the second group contains all $\sigma_{a_{i j}}$ 's, and one can prove inductively that the first group contains $\sigma_{a_{13}}, \ldots, \sigma_{a_{1 n}}$, hence the second group. 
Lemma 3.9 If $a_{i j}, a_{j k}, a_{k l}$ and $a_{i l}$ are the sides of a topological quadrangle taken in positive order and $a_{i k}, a_{j l}$ are its diagonals so that $a_{i j}, a_{j k}, a_{i k}$ and $a_{i j}, a_{j l}, a_{i l}$ are sides of topological triangles taken in positive order, then

$$
\sigma_{a_{i j}} \sigma_{a_{j l}} \sigma_{a_{i k}}=\sigma_{a_{j l}} \sigma_{a_{i k}} \sigma_{a_{k l}} .
$$

Proof This is easily obtained from the identity

$$
\begin{array}{rlrl} 
& \sigma_{1}\left(\sigma_{3}^{-1} \sigma_{2} \sigma_{3}\right)\left(\sigma_{2}^{-1} \sigma_{1} \sigma_{2}\right) & =\left(\sigma_{3}^{-1} \sigma_{2} \sigma_{3}\right)\left(\sigma_{2}^{-1} \sigma_{1} \sigma_{2}\right) \sigma_{3} \\
& \Longleftrightarrow & \sigma_{3}^{-1} \sigma_{1} \sigma_{2} \sigma_{3} \sigma_{1} \sigma_{2} \sigma_{1}^{-1} & =\sigma_{3}^{-1} \sigma_{2} \sigma_{3} \sigma_{1} \sigma_{2} \sigma_{1}^{-1} \sigma_{3} \\
\sigma_{1} \sigma_{2} \sigma_{1} \sigma_{3} \sigma_{2} \sigma_{1}^{-1} & =\sigma_{2} \sigma_{1} \sigma_{3} \sigma_{2} \sigma_{3} \sigma_{1}^{-1} \\
\sigma_{2} \sigma_{1} \sigma_{2} \sigma_{3} \sigma_{2} & =\sigma_{2} \sigma_{1} \sigma_{2} \sigma_{3} \sigma_{2}
\end{array}
$$

in the braid group $\mathrm{Br}_{4}$, which is homomorphically mapped onto the group generated by $a_{i j}, a_{j k}, a_{k l}$ and $a_{i l}$ with

$$
\sigma_{1} \mapsto \sigma_{a_{i j}}, \sigma_{2} \mapsto \sigma_{a_{j k}}, \quad \sigma_{3} \mapsto \sigma_{a_{k l}}, \quad \sigma_{2}^{-1} \sigma_{1} \sigma_{2} \mapsto \sigma_{a_{i k}}, \quad \sigma_{3}^{-1} \sigma_{2} \sigma_{3} \mapsto \sigma_{a_{j l}}
$$

Lemma 3.10 The full-twists of type $p$, resp. of type $q$ are conjugate via twists of type $a$ and $c$, resp. $b$ and $d$.

Proof Given $p_{i}, p_{i+1}$ we may apply Lemma 3.9 , since $B_{i}^{\prime}, B_{i}^{\prime \prime}, B_{i+1}^{\prime}, B_{i+1}^{\prime \prime}$ are the vertices of a topological quadrangle with alternate sides $p_{i}, p_{i+1}$ and $a_{i, i+1}, c_{i, i+1}$.

The same argument works for $q_{i}, q_{i+1}$ with $b_{i, i+1}, d_{i, i+1}$.

A similar argument works for the following:

Lemma 3.11 The twists of type $s$ (resp. $u, u^{\prime}$ or $u^{\prime \prime}$ ) are conjugate via twists of type $a, b, c, d$.

\subsection{Deducing the theorem from the propositions}

Proof of Theorem 0.2 The proof consists of three parts.

We start showing both inclusions between the braid monodromy group and the group $H$ given in the claim; then we perform the weighted count of full-twists and their inverses.

For the first two claims we use the braid monodromy factorization appearing in Theorem 3.5.

We show preliminarily that all generators of $H$ belong to the braid monodromy group of such a factorization.

All full-twists of type $p_{1}$ and $q_{1}$ belong to the braid monodromy group, by Theorem 3.5 and by our numerical assumptions. 
Then the elementary factorizations $\beta_{f, i}$ and $\beta_{g, j}$ show that also $\sigma_{a_{1 i}}, \sigma_{c_{1 i}}$, resp. $\sigma_{b_{1 l}}, \sigma_{d_{1 l}}$ belong to the braid monodromy group.

In view of Lemma 3.8 therefore all half-twists in $G$ of type $a, b, c, d$ belong to the braid monodromy group.

The elementary factorization $\beta_{f g, 1}$ guarantees that also the elements $\sigma_{s}, \sigma_{u^{\prime}}, \sigma_{u^{\prime \prime}}$ belong to the braid monodromy group.

Concerning the other full-twists of type $p$ and $q$, they are in the braid monodromy group by virtue of Lemma 3.10.

For the reverse inclusion we have to show that $H$ contains all factors of the factorization appearing in Theorem 3.5.

For the half twists of type $a, b, c, d$ this is immediate from Lemma 3.8. For the full twists of type $p, q$ it then follows from Lemma 3.10. Therefore also the half twists of type $s$ and the cubes of half twists of type $u, u^{\prime}, u^{\prime \prime}$ are in $H$ by Lemma 3.11.

Last we have to perform the weighted count of full-twists of types $p$ and $q$.

It is equal to the weighted count of intersections of $f$ with $\Phi$, resp. $g$ with $\Psi$, which in turn is simply the algebraic intersection number of each pair of divisors. Hence

$$
\begin{aligned}
& \#_{p}=(2 a, 2 b) \cdot(2 a-c, 2 b-d)=4 a b-2 a d+4 b a-2 b c \\
& \#_{q}=(2 c, 2 d) \cdot(2 c-a, 2 d-b)=4 c d-2 c b+4 d c-2 d a .
\end{aligned}
$$

\section{Proof of the conjugacy theorem}

In this last section we are going to prove that the conjugacy classes of $\sigma_{p}^{2}$ and $\sigma_{q}^{2}$ are different in the stabilised braid monodromy group $\hat{H}$; cf [10] for an alternative proof. We rely on the unicity of the "roots" $\sigma_{p}$ and $\sigma_{q}$ and on the following proposition:

Proposition 4.1 The elements $\sigma_{p}$ and $\sigma_{q}$ are not $\hat{H}$-conjugate in the braid group $G$, ie there is no $\hat{h} \in \hat{H}$ such that $\hat{h} \sigma_{p}=\sigma_{q} \widehat{h}$.

Proof The idea is to show that there is a homomorphism $\left\langle\hat{H}, \sigma_{p}, \sigma_{q}\right\rangle \rightarrow \Sigma$, such that the images of $\sigma_{p}, \sigma_{q}$ are not conjugate under the image $\hat{\Gamma}$ of $\hat{H}$.

The group $\hat{H}$ consists of liftable braids for the simple $4: 1$ covering $X_{0} \rightarrow F_{0} \cong \mathbb{P}^{1}$ branched at $4(b+d)$ points associated to the covering monodromy homomorphism $\pi_{1}\left(\mathbb{P}^{1}-\mathrm{pts}\right) \rightarrow \mathfrak{S}_{4}$, but $\sigma_{p}$ and $\sigma_{q}$ do not lift, so $\Sigma$ can not be taken to be related with this covering.

Consider instead the composed map $\pi_{1}\left(\mathbb{P}^{1}-\right.$ pts $) \rightarrow \mathfrak{S}_{4} \rightarrow \mathfrak{S}_{3}$, and the associated 3:1 covering $Y_{0} \rightarrow F_{0}$, then the elements of $\hat{H}$ and $\sigma_{p}, \sigma_{q}$ lift. 
In particular we can take $\Sigma=\operatorname{Sp}\left(H_{1}\left(Y_{0}, \mathbf{Z} / 2\right)\right)$. Since half twists map to transvections, full twist map to the identity in $\Sigma$, hence the image $\widehat{\Gamma}$ of $\hat{H}$ is the same as the image $\Gamma$ of $H$. It thus suffices to show, that the images of $\sigma_{p}$ and $\sigma_{q}$ are not conjugate under the image $\Gamma$ of $H$.

Since $\sigma_{p}$ and $\sigma_{q}$ map to transvections on vectors $v_{p}, v_{q} \in H_{1}\left(Y_{0}, \mathbf{Z} / 2\right)$ the claim follows from the following claim, which is going to be proven in Proposition 4.10:

Claim $v_{p}$ and $v_{q}$ belong to different orbits for the $\Gamma$-action on $H_{1}\left(Y_{0}, \mathbf{Z} / 2\right)$.
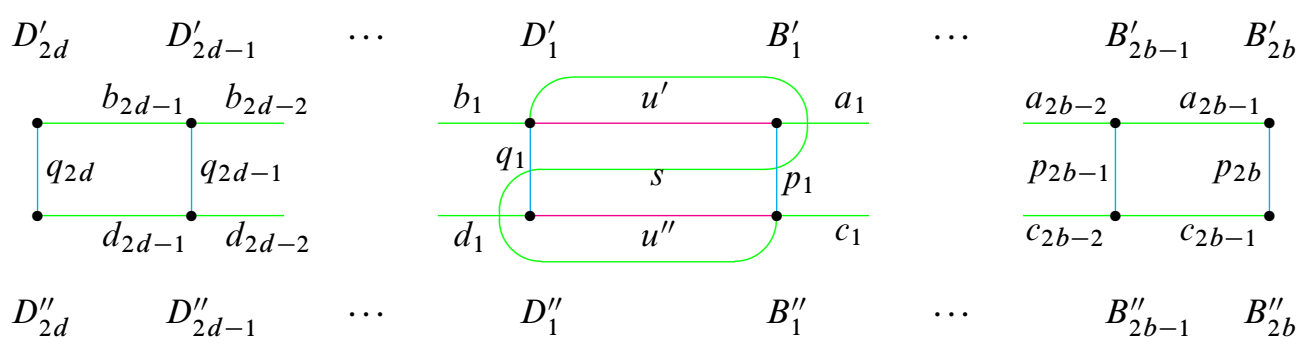

Figure 5: Isotoped generators

Let us first consider in more detail the corresponding geometrical properties of the two coverings of $F_{0}$.

The branched covering $X_{0} \rightarrow F_{0}$ is associated with a map $\pi_{1}\left(F_{0}-\left.\Delta\right|_{0}\right) \rightarrow \mathfrak{S}_{4}$. We define a further branched covering $Y_{0} \rightarrow F_{0}$ by means of the induced homomorphism $\pi_{1}\left(F_{0}-\left.\Delta\right|_{0}\right) \rightarrow \mathfrak{S}_{3}$ using the natural surjection $\mathfrak{S}_{4} \rightarrow \mathfrak{S}_{3}$ with kernel given by the Klein four-group.

For a simple 4-covering, taken an arc connecting two branch points, the minimal power of the corresponding half twist which is liftable is

(1) one, if the two associated transpositions of the local monodromies are equal,

(2) two, if they are disjoint,

(3) three, if they do not commute.

We recall from Theorem 0.2, that (1) applies for arcs of type $a, b, c, d, s$, that (2) applies for types $p, q$ and (3) applies for type $u$.

Considering now the associated simple 3 -covering we note that each arc in the base between branch points has a preimage, which consists of either

(i) a single arc containing both ramification points, or

(ii) a cycle containing both ramification points and a residual arc, 
depending on whether the local monodromies with respect to a base point on the arc yield two different or two equal transpositions.

In fact since every branch point corresponds to a transposition in monodromy, the product of two can only be a 3-cycle or trivial in case of a simple branched cover of degree 3 .

Since in case (ii) the half twist on the arc lifts to a diffeomorphism of the 3-covering and in case (i) only the cube of the half twist does, we have the following implications:

$\begin{array}{llll}\begin{array}{l}\text { case (1) for } X_{0} \\ \text { case (2) for } X_{0}\end{array} \begin{array}{ll}\text { case (ii) for } Y_{0} \\ \text { case (ii) for } Y_{0}\end{array} \quad \begin{array}{l}\text { (If the cube and the square lift, } \\ \text { then so does the half twist itself.) }\end{array} \\ \text { case (3) for } X_{0} \Longrightarrow & \text { case (i) for } Y_{0}\end{array}$

In particular only the arcs $u_{i}$ belong to the first case, while all others, of types $a, b, c, d, p, q, s$, belong to the second case.

For the following discussion we need cycles on $Y_{0}$ and their intersection pairings. We denote by $\tilde{v}$ the cycle in the preimage of an arc $v$ in the second class.

Lemma 4.2 For the $\mathbb{Z} / 2$-valued intersection pairing $\langle$,$\rangle of cycles on Y_{0}$,

$$
\left\langle\widetilde{v}_{1}, \widetilde{v}_{2}\right\rangle \equiv_{2} \#\left(v_{1} \cap v_{2}\right),
$$

if $v_{1} \neq v_{2}$ and $v_{1}, v_{2} \in\left\{a_{i}, b_{i}, c_{l}, d_{l}, p_{j}, q_{j}, s\right\}$.

Proof It suffices to prove that there is a bijection of points of $\widetilde{v}_{1} \cap \widetilde{v}_{2}$ with points of $v_{1} \cap v_{2}$ given by the restriction of the projection map.

Each branch point has two preimages, a ramification point and a point, where the map is unramified, but it is always the ramification point which belongs to a cycle in the preimage of any arc from that branch point. A pair of cycles $\widetilde{v}_{1}, \widetilde{v}_{2}$ meets in the ramification point if $v_{1}, v_{2}$ meet in the branch point. Hence two arcs share a branch point if and only if they share the corresponding ramification point.

All ordinary points have three preimages. But a cycle in the preimage of an arc contains either none or two of those. Hence either one or two of the preimages of an intersection between two arcs are shared by their corresponding cycles on $Y_{0}$.

Observe that the only pairs of arcs which meet in a point which is not a branch point are $s$ and one of $a_{1}, d_{1}, p_{1}, q_{1}$.

First we consider the four local monodromies with respect to a base point at the intersection of $s$ and $p_{1}$ along their segments. These monodromies are identical if and 
only if the cycles $\tilde{s}$ and $\tilde{p}_{1}$ meet in two points. If so, we could conclude by isotopy that the two monodromies associated to $u^{\prime}$ must be the same. Since on the contrary $u^{\prime}$ belongs to class i), we have proved that $\# \widetilde{s} \cap \tilde{p}_{1}=1$.

For $s$ and $a_{1}$ we have the same argument and we conclude for $s$ and $q_{1}$ resp. $d_{1}$ by symmetry.

Lemma 4.3 A basis of $H_{1}(Y, \mathbb{Z} / 2)$ is given by

$$
\tilde{a}_{3}, \ldots, \tilde{a}_{2 b-1}, \tilde{p}_{2 b}, \tilde{c}_{2 b-1}, \ldots, \tilde{c}_{1}, \tilde{s}, \tilde{b}_{1}, \ldots, \tilde{b}_{2 d-1}, \tilde{q}_{2 d}, \tilde{d}_{2 d-1}, \ldots, \tilde{d}_{2} .
$$

Proof From the previous lemma we can deduce that the intersection matrix for the given elements is tridiagonal with diagonal entries 0 and entries in the secondary diagonals all equal to 1 . Hence the intersection form on these elements is nondegenerate and thus the elements are linearly independent.

On the other hand by the Riemann-Hurwitz formula their number

$$
4 b+4 d-4=2 b-3+1+2 b-1+1+2 d-1+1+2 d-2
$$

equals the dimension of $H_{1}(Y, \mathbb{Z} / 2)$ since

$$
\begin{aligned}
e\left(Y_{0}\right) & =3 \cdot 2-4 b-4 d \\
\Longrightarrow \quad b_{1}\left(Y_{0}\right) & =2-e=4 b+4 d-4 .
\end{aligned}
$$

Lemma 4.4 There is a quadratic form $\mathbf{q}$ on $H_{1}$ such that

(1) $\mathbf{q}(w)=1$ for all elements of the basis given in Lemma 4.3,

(2) the intersection pairing coincides with the induced symmetric form:

$$
\left\langle\widetilde{v}_{1}, \tilde{v}_{2}\right\rangle=\left\langle\widetilde{v}_{1}, \widetilde{v}_{2}\right\rangle_{\mathbf{q}}:=\mathbf{q}\left(\widetilde{v}_{1}+\widetilde{v}_{2}\right)+\mathbf{q}\left(\widetilde{v}_{1}\right)+\mathbf{q}\left(\widetilde{v}_{2}\right) .
$$

Proof Obviously the quadratic form associated with the matrix $Q$ meets both requirements, if the diagonal element are given by $Q_{i i}=1$ and the off-diagonal element are given by $Q_{i j}=0$ if $i>j$ and $Q_{i j}=\left\langle\widetilde{v}_{i}, \widetilde{v}_{j}\right\rangle$ if $i<j$.

We consider the subgroup $\tilde{H}$ of the braid group generated by the braid monodromy group and by the half twists on the arcs $p_{j}, q_{j}$.

Lemma 4.5 All elements of $\tilde{H}$ considered as isotopy classes of homeomorphisms of $\left(F_{0},\left.\Delta\right|_{0}\right)$ can be lifted to isotopy classes of homeomorphisms of $Y$. 
Proof An element is liftable if and only if its induced map on the natural homomorphism is the identity. Since elements of the braid monodromy group stabilise the natural homomorphism to $\mathfrak{S}_{4}$, they stabilise also its composition with $\mathfrak{S}_{4} \rightarrow \mathfrak{S}_{3}$.

Half-twists on $\operatorname{arcs} p_{i}$ and $q_{i}$ do not stabilise the natural homomorphism to $\mathfrak{S}_{4}$, but they exchange disjoint transpositions. However such pairs are identified under $\mathfrak{S}_{4} \rightarrow \mathfrak{S}_{3}$, hence also the additional elements lift.

We consider next the action of $\Gamma$ via the mapping class group of $Y$ on the first homology of $Y$ with $\mathbb{Z} / 2$ coefficients preserving the natural symplectic form.

Note that the preimage in $Y$ of some arc in $F_{0}$ contains a simple closed curve if and only if the half-twist on that arc lifts to $Y$.

Lemma 4.6 The image of $\tilde{H}$ in $\operatorname{Sp} H_{1}(Y ; \mathbb{Z} / 2)$ is generated by the symplectic transvection on all the classes of $H_{1}$ which are represented by simple closed curves mapping to the arcs $a, b, c, d, q, p, s$.

Proof Since $Y \rightarrow F$ is a simple triple cover, any arc either lifts to an arc in $Y$ or the union of an arc with a simple closed curve. In the first case the third power of the corresponding half twist lifts, but its lift is the half twist on the preimage and therefore isotopic to the identity.

In the second case the corresponding half twist lifts to the product of the half twist on the arc and a Dehn twist on the circle. The former is isotopic to the identity but the latter acts by a symplectic transvection by the class of the circle.

Hence it suffices to take all transvection associate to circles in the lifts of the arcs needed to generated $\Gamma$.

Lemma 4.7 If $\mathbf{q}$ is a quadratic form on a $\mathbb{Z} / 2-$ vector space such that $\langle,\rangle_{\mathbf{q}}$ is the corresponding symmetric bilinear form, then $\mathbf{q}(w)=1$ implies $\mathbf{q}\left(T_{w} z\right) \equiv_{2} \mathbf{q}(z)$.

Proof By assumption either $\langle w, z\rangle_{\mathbf{q}}$ or $\langle w, z\rangle_{\mathbf{q}}+\mathbf{q}(w)$ is even, hence

$$
\begin{aligned}
\mathbf{q}\left(T_{w} z\right) & \equiv{ }_{2} \mathbf{q}\left(z+\langle w, z\rangle_{\mathbf{q}} w\right) \\
& \equiv{ }_{2} \mathbf{q}(z)+\mathbf{q}\left(\langle w, z\rangle_{\mathbf{q}} w\right)+\left(z,\langle w, z\rangle_{\mathbf{q}} w\right) \\
& \equiv{ }_{2} \mathbf{q}(z)+\langle w, z\rangle_{\mathbf{q}}\left(\mathbf{q}(w)+\langle w, z\rangle_{\mathbf{q}}\right) \\
& \equiv{ }_{2} \mathbf{q}(z) .
\end{aligned}
$$

Lemma 4.8 If $\mathbf{q}(w) \equiv_{2} 0$ and $\langle w, z\rangle_{\mathbf{q}} \equiv_{2} 1$, then $\mathbf{q}\left(T_{w} z\right) \equiv_{2} \mathbf{q}(z)+1$.

Proof By the same computation as above but with $\langle w, z\rangle_{\mathbf{q}}$ and $\langle w, z\rangle_{\mathbf{q}}+\mathbf{q}(w)$ both odd. 
Proposition 4.9 The transvections associated to $q_{2 b}$ and $p_{2 d}$ are not conjugate under the group $\Gamma$ generated by the transvections on the elements $a_{i}, b_{i}, c_{i}, d_{i}, s$.

Proof We work with the basis of $H_{1}$ given in Lemma 4.3

$\tilde{a}_{3}, \tilde{a}_{4}, \ldots, \tilde{a}_{2 b-1}, \tilde{p}_{2 b}, \tilde{c}_{2 b-1}, \ldots, \tilde{c}_{2}, \tilde{c}_{1}, \tilde{s}_{1} \tilde{b}_{1}, \tilde{b}_{2}, \ldots, \tilde{b}_{2 d-1}, \tilde{q}_{2 d}, \tilde{d}_{2 d-1}, \ldots, \tilde{d}_{3}, \tilde{d}_{2}$

and the quadratic form $\mathbf{q}: H_{1} \rightarrow \mathbb{Z} / 2$ of Lemma 4.4 , which is nontrivial on all basis elements.

By straightforward computation by looking at the intersection number, we get for the remaining elements $a_{1}, a_{2}, d_{1}$ :

$$
\begin{aligned}
\tilde{a}_{1}= & \tilde{a}_{3}+\tilde{a}_{5}+\cdots+\tilde{a}_{2 b-1}+\tilde{c}_{2 b-1}+\cdots+\tilde{c}_{3}+\tilde{c}_{1} \\
\tilde{a}_{2}= & \tilde{a}_{4}+\tilde{a}_{6}+\cdots+\tilde{a}_{2 b-2}+\tilde{p}_{2 b}+\tilde{c}_{2 b-2}+\cdots+\tilde{c}_{4}+\tilde{c}_{2}+\tilde{s} \\
& +\tilde{b}_{2}+\tilde{b}_{4}+\cdots+\tilde{b}_{2 d-2}+\tilde{q}_{2 d}+\tilde{d}_{2 d-2}+\cdots+\tilde{d}_{4}+\tilde{d}_{2} \\
\tilde{d}_{1}= & \tilde{b}_{1}+\tilde{b}_{3}+\cdots+\tilde{b}_{2 d-1}+\tilde{d}_{2 d-1}+\cdots+\tilde{d}_{5}+\tilde{d}_{3} \\
\mathbf{q}\left(a_{1}\right)= & 1, \quad \mathbf{q}\left(a_{2}\right)=0, \quad \mathbf{q}\left(d_{1}\right)=1
\end{aligned}
$$

Let us denote by $\delta_{p}$ the element of the dual basis, which evaluates nontrivial on $p_{2 b}$ and let us introduce a function

$$
\rho: H_{1}(Y, \mathbb{Z} / 2) \rightarrow \mathbb{Z} / 2, \quad x \mapsto \delta_{p}(x)+\mathbf{q}(x) .
$$

Next we note that this function is invariant under the action of $\Gamma$, since it is under the transvections associated to any one of the elements $w$ given in the statement of this proposition:

$$
\begin{aligned}
\left.\rho\left(T_{w} x\right)\right) & =\delta_{p}\left(T_{w} x\right)+\mathbf{q}\left(T_{w} x\right) \\
& =\delta_{p}(x)+\langle x, w\rangle \delta_{p}(w)+\mathbf{q}(x)+\mathbf{q}(\langle x, w\rangle w)+(x,\langle x, w\rangle w) \\
& =\delta_{p}(x)+\mathbf{q}(x)+\langle x, w\rangle\left(\delta_{p}(w)+\mathbf{q}(w)+\langle x, w\rangle\right) \\
& =\rho(x)
\end{aligned}
$$

The product on the right in the second but last step has factors of either parity, since $\delta_{p}(w)+\mathbf{q}(w)=1$ due to

$$
\delta_{p}(w)=\left\{\begin{array}{ll}
1 & \text { for } w=a_{2}, \\
0 & \text { else, }
\end{array} \quad \mathbf{q}(w)= \begin{cases}0 & \text { for } w=a_{2}, \\
1 & \text { else. }\end{cases}\right.
$$

Since $\rho(q)=\mathbf{q}(q)+\delta_{p}(q)=1+0=1$ and $\rho(p)=\mathbf{q}(p)+\delta_{p}(p)=1+1 \equiv_{2} 0$ the two elements $q, p$ are not contained in the same $\Gamma$-orbit. 
Proposition 4.10 No full twist on any arc $p_{i}$ is conjugate in the braid monodromy group to a full twist on any arc $q_{j}$.

Proof Suppose to the contrary that there is a pair $p_{i}, q_{j}$ such that the corresponding full twist are conjugate under some $\beta$ from the braid monodromy group.

Then $\beta$ maps $p_{i}$ to $q_{j}$ or vice versa. In particular also the half-twists on $p_{i}$ and $q_{j}$ are conjugate under $\beta$ in the braid monodromy group.

But then the transvections associated to $p$ and $q$ are conjugate under the symplectic transformation associated to $\beta$. That is in contradiction to what we proved earlier, so our claim must be true.

Acknowledgements The present research was performed in the realm of the Forschergruppe 790 "Classification of algebraic surfaces and compact complex manifolds" of the DFG (DeutscheForschungsGemeinschaft).

The results of the paper were announced in a workshop which took place in October 2008 during the special trimester "Groups in algebraic geometry" at the Centro De Giorgi of the Scuola Normale di Pisa.

\section{References}

[1] D Auroux, S K Donaldson, L Katzarkov, M Yotov, Fundamental groups of complements of plane curves and symplectic invariants, Topology 43 (2004) 1285-1318 MR2081427

[2] D Auroux, L Katzarkov, Branched coverings of $\mathbf{C P}^{2}$ and invariants of symplectic 4-manifolds, Invent. Math. 142 (2000) 631-673 MR1804164

[3] D Auroux, L Katzarkov, A degree doubling formula for braid monodromies and Lefschetz pencils, Pure Appl. Math. Q. 4 (2008) 237-318 MR2400878

[4] D Auroux, I Smith, Lefschetz pencils, branched covers and symplectic invariants, from: "Symplectic 4-manifolds and algebraic surfaces", (F Catanese, G Tian, editors), Lecture Notes in Math. 1938, Springer, Berlin (2008) 1-53 MR2441411

[5] F Catanese, On the moduli spaces of surfaces of general type, J. Differential Geom. 19 (1984) 483-515 MR755236

[6] F Catanese, Automorphisms of rational double points and moduli spaces of surfaces of general type, Compositio Math. 61 (1987) 81-102 MR879190

[7] F Catanese, Symplectic structures of algebraic surfaces and deformation arXiv: math.AG/0207254 
[8] F Catanese, Differentiable and deformation type of algebraic surfaces, real and symplectic structures, from: "Symplectic 4-manifolds and algebraic surfaces", (F Catanese, G Tian, editors), Lecture Notes in Math. 1938, Springer, Berlin (2008) 55-167 MR2441412

[9] F Catanese, Canonical symplectic structures and deformations of algebraic surfaces, Commun. Contemp. Math. 11 (2009) 481-493 MR2538209

[10] F Catanese, M Lönne, B Wajnryb, Moduli spaces of surfaces and monodromy invariants, from: "Proceedings of the Gökova Geometry-Topology Conference 2009", (S Akbulut, D Auroux, T Önder, editors), Int. Press, Somerville, MA (2010) 58-98 MR2655304

[11] F Catanese, B Wajnryb, The 3-cuspidal quartic and braid monodromy of degree 4 coverings, from: "Projective varieties with unexpected properties", (C Ciliberto, A V Geramita, B Harbourne, R M Miró-Roig, K Ranestad, editors), de Gruyter, Berlin (2005) 113-129 MR2202250

[12] F Catanese, B Wajnryb, Diffeomorphism of simply connected algebraic surfaces, J. Differential Geom. 76 (2007) 177-213 MR2330412

[13] S K Donaldson, The Seiberg-Witten equations and 4-manifold topology, Bull. Amer. Math. Soc. (N.S.) 33 (1996) 45-70 MR1339810

[14] S K Donaldson, Lefschetz pencils on symplectic manifolds, J. Differential Geom. 53 (1999) 205-236 MR1802722

[15] C Ehresmann, Sur les espaces fibrés différentiables, C. R. Acad. Sci. Paris 224 (1947) 1611-1612 MR0020774

[16] M H Freedman, The topology of four-dimensional manifolds, J. Differential Geom. 17 (1982) 357-453 MR679066

[17] R Friedman, J W Morgan, Algebraic surfaces and 4-manifolds: some conjectures and speculations, Bull. Amer. Math. Soc. (N.S.) 18 (1988) 1-19 MR919651

[18] R E Gompf, A new construction of symplectic manifolds, Ann. of Math. (2) 142 (1995) 527-595 MR1356781

[19] R E Gompf, A I Stipsicz, 4-manifolds and Kirby calculus, Graduate Studies in Math. 20, Amer. Math. Soc. (1999) MR1707327

[20] J González-Meneses, B Wiest, On the structure of the centralizer of a braid, Ann. Sci. École Norm. Sup. (4) 37 (2004) 729-757 MR2103472

[21] A Kas, On the handlebody decomposition associated to a Lefschetz fibration, Pacific J. Math. 89 (1980) 89-104 MR596919

[22] M Lönne, Fundamental groups of projective discriminant complements, Duke Math. J. 150 (2009) 357-405 MR2569617

[23] M Lönne, Braid monodromy of some Brieskorn-Pham singularities, Internat. J. Math. 21 (2010) 1047-1070 MR2679383 
[24] M Manetti, On some components of moduli space of surfaces of general type, Compositio Math. 92 (1994) 285-297 MR1286128

[25] M Manetti, Iterated double covers and connected components of moduli spaces, Topology 36 (1997) 745-764 MR1422433

[26] M Manetti, On the moduli space of diffeomorphic algebraic surfaces, Invent. Math. 143 (2001) 29-76 MR1802792

[27] B Moishezon, Stable branch curves and braid monodromies, from: "Algebraic geometry (Chicago, Ill., 1980)", (A Libgober, P Wagreich, editors), Lecture Notes in Math. 862, Springer, Berlin (1981) 107-192 MR644819

[28] B Moishezon, Algebraic surfaces and the arithmetic of braids. I, from: "Arithmetic and geometry, Vol. II", (M Artin, J Tate, editors), Progr. Math. 36, Birkhäuser, Boston (1983) 199-269 MR717613

[29] B Moishezon, The arithmetic of braids and a statement of Chisini, from: "Geometric topology (Haifa, 1992)", (C Gordon, Y Moriah, B Wajnryb, editors), Contemp. Math. 164, Amer. Math. Soc. (1994) 151-175 MR1282761

[30] B Moishezon, M Teicher, Finite fundamental groups, free over $\mathbf{Z} / c \mathbf{Z}$, for Galois covers of $\mathbf{C P}^{2}$, Math. Ann. 293 (1992) 749-766 MR1176029

FC, ML: Mathematisches Institut, Lehrstuhl Mathematik VIII, Universität Bayreuth Universitätsstraße 30, D-95447 Bayreuth, Germany

BW: Department of Mathematics, Rzeszów University of Technology ul W Pola 2, 35-959 Rzeszów, Poland

Fabrizio.Catanese@uni-bayreuth.de, Michael.Loenne@uni-bayreuth.de, dwajnryb@prz.edu.pl

http://www . mathe8.uni-bayreuth.de/Personen/Catanese.html

Proposed: Ronald Fintushel

Seconded: Joan Birman, Ron Stern
Received: 12 October 2009 Accepted: 8 November 2010 